\title{
Oligopoly Games under Asymmetric Costs and an Application to Energy Production
}

\author{
Andrew Ledvina* $\quad$ Ronnie Sircar ${ }^{\dagger}$
}

First version: July 2011; revised January 2012 and March 2012

\begin{abstract}
Oligopolies in which firms have different costs of production have been relatively understudied. In contrast to models with symmetric costs, some firms may be inactive in equilibrium. (With symmetric costs, the results trivialize to all firms active or all firms inactive.) We concentrate on the linear demand structure with constant marginal but asymmetric costs. In static one-period models, we compare the number of active firms, i.e. the number of firms producing a positive quantity in equilibrium, across four different models of oligopoly: Cournot and Bertrand with homogeneous or differentiated goods. When firms have different costs, we show that, for fixed good type, Cournot always results in more active firms than Bertrand. Moreover, with a fixed market type, differentiated goods result in more active firms than homogeneous goods. In dynamic models, asymmetric costs induce different entry times into the market. We illustrate with a model of energy production in which multiple producers from costly but inexhaustible alternative sources such as solar or wind compete in a Cournot market against an oil producer with exhaustible supply.
\end{abstract}

JEL classification: C72; D43; L11; L22

Keywords: Oligopolies; Bertrand model; Cournot model; Differentiated goods; Dynamic games; Exhaustible resources.

\section{Introduction}

The main focus of this paper is to study the number of firms in an oligopoly who actively produce in equilibrium when they have different costs of production. Asymmetric costs are commonplace in markets of large and small producers (for example a major chain such as Barnes \& Noble in the U.S. vs. an independent local bookstore), where the larger firm can achieve economies of scale. In energy markets, producers from different fuels and technologies have widely different costs of production: for example, oil and coal sources are much cheaper to produce from than renewables such as solar

*ORFE Department, Princeton University, Sherrerd Hall, Princeton NJ 08544; aledvina@princeton.edu. Work partially supported by NSF grant DMS-0739195.

†ORFE Department, Princeton University, Sherrerd Hall, Princeton NJ 08544; sircar@princeton.edu. Work partially supported by NSF grant DMS-0807440. 
or hydropower. However, most analyses assume symmetric costs, or restricted cost structures that guarantee all firms are active in equilibrium. Whether a firm produces a positive quantity in an equilibrium is an important question in the study of oligopolistic competition because it can be used to explain market variety as a cost to the consumer.

We first focus on both the Cournot and Bertrand static games within which we have two cases: when the goods are homogeneous, and when they are differentiated. We will show that, within a fixed type of market, Cournot or Bertrand, differentiated goods result in more active firms in Nash equilibrium than homogeneous goods. These results depend crucially on cost asymmetries between the firms, as with symmetric costs the results trivialize to all firms active or all firms inactive. Our results, e.g. Theorem 4.1, partially explain the relative differences to consumers between these two types of markets, as consumers have more choice in Cournot markets but with higher prices, whereas they have less choice in Bertrand markets (as the number of firms in equilibrium is less) but they are compensated for this fact by reduced prices.

Then we show the impact of cost differences in a dynamic Cournot game between energy producers, one of which has a cheap exhaustible source such as oil, while the others have inexhaustible costly technologies, such as solar or wind. Here, the Markov perfect equilibrium is characterized by nonlinear differential equations which we can solve to determine the entry points of the alternative producers into the market. We show how entry leads to jumps in the rate of change over time of the equilibrium energy price and derive a modified Hotelling's rule for exhaustible resources under oligopolistic competition (Propositions 5.6 and 5.7).

\section{$1.1 \quad$ Background}

The study of non-cooperative oligopolistic competition originated with the seminal work of Cournot [6]. His original model assumes firms choose quantities of a homogeneous good to supply and then receive profit based on the single market price as determined through a linear inverse demand function of the aggregate market supply. Moreover, marginal costs of production were assumed constant and equal across firms. Throughout this paper, we also assume constant marginal costs, however we drop the cost symmetry assumption. The result of Cournot's analysis is what one would expect: with equal marginal costs across firms, every firm chooses the same quantity to supply and the market price is above cost by an amount that is inversely proportional to one plus the number of firms in the market. Hence, as the number of firms tends to infinity, the price approaches marginal cost, but with a finite number of firms, prices are above cost and firms earn positive profits.

Following this, Bertrand [3] argued to change the strategic variable from quantity to price. Firms producing a homogeneous good were assumed to set prices and produce to meet any demand of the market. As the goods are homogeneous, there can only be one market price, as observed in the Cournot model above, and therefore only the firm quoting the minimum price receives any demand. This feature of "winner takes all" leads to very harsh and contradictory results relative to common market observations. If all firms have equal cost, then as long as there are two or more firms in the market, all firms price at cost and have zero profit. This perfectly competitive outcome differs substantially from the Cournot outcome and is commonly referred to as the Bertrand paradox. To paraphrase from [21], the Bertrand model results in perfect competition in all cases besides monopoly, which is unrealistic in most settings, leading one to conclude that the correct set-up leads to the wrong result. On the other hand, as most firms seem to set their prices, not their 
quantities, many economists have argued that the Cournot model gives the right answer for the wrong reason.

Since these two original papers, there has been much interest in modifying these models in various ways to obtain more realistic results. Furthermore, there is a significant literature comparing the two modes of competition when some of the assumptions in the original papers are altered. These modifications are usually brought about to reconcile the Bertrand paradox. If one considers constraints on the capacity of the firms, see Edgeworth [8], then the result of the Bertrand model can be brought closer to that of the Cournot model. Kreps and Scheinkman [18] use capacity constraints to obtain the Cournot outcome from Bertrand competition. As such, the Bertrand model cannot really be thought of as complete without more realistic assumptions.

One of the original assumptions that is commonly not satisfied is that goods are homogeneous. This leads one to consider differentiated goods, which can mean either substitute or complementary goods, although we concentrate on the case of substitute goods. The original work in this area is Hotelling [16], where consumers were assumed to associate a cost of travel depending on the location of the firms, thereby differentiating their otherwise identical goods based on the location of the firms relative to the location of the consumer. For classical results on differentiated goods and other models of oligopoly, we refer to the books by Friedman [10] and Vives [24].

\section{$1.2 \quad$ Asymmetric Costs}

Very often, modifications of the original models are made under the assumption of symmetric marginal costs across firms. When one considers an asymmetric cost structure, the issue of the number of firms who are active in an equilibrium becomes crucially important. This fact is often overlooked or assumed away in studies of asymmetric cost, see for example [17] and [1]. As we will see though, it is not always the case that inactive firms can simply be ignored - their presence may affect equilibrium quantities and prices. We refer also to [5] and [22] for analysis of consumer surplus under Bertrand and Cournot modes of competition, but again with symmetric (and zero) costs.

The issue of asymmetric cost in oligopolies has been addressed before. In the work of Singh and Vives [23], they consider a game in which firms in a differentiated duopoly can choose to offer either price or quantity contracts to consumers. Essentially this allows firms to select either Bertrand or Cournot competition. They show for substitute goods that it is always a dominant strategy for both firms to choose the quantity competition. However, they derive their results under the assumption that both firms are active in equilibrium. In a related work, Zanchettin [25] compares prices and outputs in a duopoly with linear demand where the firms can have asymmetric costs and the goods are differentiated. The main idea is to remove the assumption that both firms are active in equilibrium and to consider the effect of this on prices and outputs. The main results of this paper are that prices are lower in Bertrand compared to Cournot, however outputs of both firms may not always be greater in the Bertrand market relative to the Cournot market. Furthermore, industry profits can be higher in Bertrand than in Cournot for certain parameter values. This work is closest to ours although, as it is a duopoly model, the possible number of active firms is restricted to one or two which simplifies the analysis considerably. Furthermore, additional asymmetries are tractable in a duopoly that are essentially unworkable in an $N$-firm oligopoly. 
Static Cournot/Bertrand games also arise as an intermediary in their dynamic counterparts, where asymmetric shadow costs encode firms' scarcity values as their resources or capacities deplete. See [13] for a Cournot dynamic game with exhaustible resources and [19] for a dynamic Bertrand game in which firms of different sizes begin competing with asymmetric lifetime capacities. Study of these leads to the issue of blockading - where some firms may be inactive due to high shadow costs until the resources of their competitors fall (and their shadow costs rise) accordingly. Hence, a thorough analysis of the activity levels in the static Cournot and Bertrand games is needed, which is the purpose of Sections 3-4. The number of active firms is generally studied in terms of entry and exit from a market in a dynamic setting, see for example [9] and [2]. We compute explicitly the entry points as a function of the asymmetric costs in a dynamic Cournot energy production model in Section 5.

\section{Models of Oligopolistic Competition}

We start with a market that has $N \geq 1$ firms. Let $q_{i}$ be the quantity produced by Firm $i$, and similarly let $p_{i}$ be the price charged for their respective good. Dropping the subscript, we denote by $p$ and $q$ the vector of all prices or quantities, respectively. We define $Q=\sum q_{i}$ and $P=\sum p_{i}$ as the aggregate quantity and price, respectively.

\subsection{Substitutability of Goods}

We focus on two types of markets: those where the goods being sold are not identical, and those where the goods are identical.

Differentiated goods means that the goods are not perfect substitutes for one another. Each firm can in principle then receive demand even if they are not the lowest price firm or the firm producing the highest quantity.

Homogeneous goods means that the goods sold by the different firms are perfect substitutes for one another. Therefore, only one price can prevail in the market. In the case of firms choosing quantities, this does not add undue burden at all, as it simply implies that the price a firm receives will depend on the aggregate market supply and their profit will then depend on that price and their chosen quantity. The case of firms setting prices is more complicated. Each firm can offer a price, but only one price can ultimately prevail in the market as goods are deemed identical. Thus, only the firms setting the lowest price will receive demand from the market. The rest can post a price, but as they will not receive demand at this higher price, it is irrelevant. The only issue that arises is in a tie for the lowest price. To accommodate this important case, one must define precisely how the lowest price firms share demand in equilibrium. These rules are commonly referred to as sharing rules and can have important equilibrium consequences, see for example [14]. We shall make explicit in what follows which sharing rule we use and our reason for doing so. 


\section{$2.2 \quad$ Linear Oligopolies}

We concentrate on a linear demand specification in order to present explicit calculations, particularly for the number of active firms in equilibrium. However, the existence of an equilibrium in each of the four models under asymmetric costs can be established in more general settings: for the homogeneous Cournot case, see [13, Section 2], and [19, Section 2] for the differentiated Bertrand case.

We derive demand from the behavior of a representative consumer with the following quadratic utility function:

$$
U(q)=\alpha \sum_{i=1}^{N} q_{i}-\frac{1}{2}\left(\beta \sum_{i=1}^{N} q_{i}^{2}+\gamma \sum_{\substack{i=1 \\ j \neq i}}^{N} \sum_{\substack{j=1 \\ j}}^{N} q_{i} q_{j}\right), \quad \alpha, \beta>0 .
$$

Our representative consumer solves the utility maximization problem

$$
\max _{q \in \mathbb{R}_{+}^{N}} U(q)-\sum_{i=1}^{N} p_{i} q_{i}
$$

from which we can derive the inverse demand functions for the firms as

$$
p_{i}(q)=\frac{\partial U}{\partial q_{i}}=\alpha-\beta q_{i}-\gamma \sum_{j \neq i} q_{j}, \quad i=1, \cdots, N . \quad \text { Differentiated Cournot }
$$

This gives actual quantities provided $p_{i}>0$ for all $i$. In the case that the quantities result in any of the prices not being positive, we must remove the individual firm with the highest quantity from the system and then consider a market with one less firm. If any of the prices are still not positive, then we repeat this procedure, one firm at a time, until we have a market with positive prices for all firms.

The parameter $\gamma$ can be positive, negative or zero depending on whether the goods are substitutes, complements or independent.

Definition 2.1. Within the linear demand set-up, the following concepts can be characterized:

- Independent goods $\quad \gamma=0$;

- Substitute goods $\quad \gamma>0$;

- Differentiated goods $\quad \gamma<\beta$;

- Homogeneous goods $\quad \gamma=\beta$.

We will not deal with the case of complementary goods, which corresponds to $\gamma<0$. The quantity $\gamma / \beta$ expresses the degree of product differentiation, which ranges from zero (independent goods) to one (perfect substitutes, or homogeneous goods).

When $\gamma<\beta$, System (2) can be inverted to obtain

$$
q_{i}(p)=a_{N}-b_{N} p_{i}+c_{N} \sum_{j \neq i} p_{j}, \quad i=1, \cdots, N, \quad \text { Differentiated Bertrand }
$$


where for $1 \leq n \leq N$, we define

$$
a_{n}=\frac{\alpha}{\beta+(n-1) \gamma}, \quad b_{n}=\frac{\beta+(n-2) \gamma}{(\beta+(n-1) \gamma)(\beta-\gamma)}, \quad c_{n}=\frac{\gamma}{(\beta+(n-1) \gamma)(\beta-\gamma)} .
$$

As in the case of inverse demands, System (3) gives the actual quantities if they are all strictly positive. If some of them are not positive, then we consider the case where the firms are ordered by price such that $p_{1} \leq p_{2} \leq \cdots \leq p_{N}$. Then, we remove the price and quantity of firm $N$ from the utility function and repeat the above procedure. If some remain that are not positive, we continue removing firms in this manner one at a time until we have only firms with positive quantities remaining. The equivalent manner to remove them at the level of the demand functions is to again consider the firms ordered by price, and to remove the price and quantity of Firm $N$ from the system and use Expression (4) with $n$ equal to $N-1$. If firms still remain with negative quantity in this reduced demand system, then we again continue this procedure one firm at a time until only firms with positive quantity remain. If firms are not ordered by price, we can re-label them for this procedure and then restore their correct labels once the demand system is determined. For differentiated goods, System (2) gives the inverse demand functions relevant to the Cournot market, and System (3) gives the demand functions relevant to the Bertrand market.

For homogeneous goods, we need to consider the limit of the utility function in Equation (1) when $\gamma=\beta$. This is no problem for the inverse demand functions and we arrive at

$$
p_{i}^{H}(q)=\alpha-\beta \sum_{j=1}^{N} q_{j}, \quad i=1, \cdots, N, \quad \text { Homogeneous Cournot }
$$

where the $H$ stands for Homogeneous, which is to distinguish this inverse demand function from that expressed in System (2). This is the same price for all of the firms and it depends only on the aggregate supply in the market. The difficulty with the Bertrand market is that we cannot invert this relationship.

The issue that arises in the Bertrand case can best be presented with a two-firm duopoly example. If we suppose $p_{1}$ is fixed and $p_{2}=p_{1}+\epsilon$ then $q_{2}(p)=a_{2}-\left(b_{2}-c_{2}\right) p_{1}-b_{2} \epsilon$, and $q_{1}=a_{2}-\left(b_{2}-c_{2}\right) p_{1}+c_{2} \epsilon$. As $\gamma$ approaches $\beta$, we have $\left(b_{2}-c_{2}\right) \rightarrow \infty$. Thus, for $\gamma$ sufficiently close to $\beta$ we will have $q_{2}<0$. However, when this occurs, the demand function for Firm 1 changes in a consistent manner to reflect the fact that Firm 2 is out of the market. This is accomplished by using Expression (4) with $n=1$. If $p_{1}=p_{2}=p$, then we have from System (5) that $\left(q_{1}+q_{2}\right)=\frac{\alpha}{\beta}-\frac{1}{\beta} p$. In other words, the quantities of the two firms must add up to the quantity that a single firm would receive if they set the lowest price alone. Furthermore, as firms are distinguishable only by the prices they set, equal prices must imply they get equal demand. Moreover, as we have established what the sum of their demands is, we know that each gets an equal share of this demand. In this way we obtain

$$
q_{i}^{H}(p)=\frac{\left(\frac{\alpha}{\beta}-\frac{1}{\beta} p_{i}\right)}{\#\left\{k: p_{k} \leq p_{j} \forall j\right\}} \mathbb{1}_{\left\{p_{i}=\min (p)\right\}} . \quad \text { Homogeneous Bertrand }
$$

For an $N$-firm oligopoly, the procedure for determining demand is identical as that above, where firms are removed from the demand system one-by-one as their quantities go negative. We thus arrive at the same conclusion regardless of the number of firms. This is the classical "winner-takeall" Bertrand model with monopoly demand $\frac{\alpha}{\beta}-\frac{1}{\beta} p$. 


\section{Nash Equilibria of the Four Static Games}

We assume that each firm has constant marginal cost, which we denote by $s_{i}$ for Firm $i$. We denote by $s$ the vector of such costs. Our main goal is to demonstrate how cost asymmetries, coupled with the degree of product differentiation and the mode of competition, affect the structure of the market. We begin with static Cournot and Bertrand games, and in Section 5, we will study a dynamic Cournot game.

Each firm chooses its price or quantity to maximize profit in a non-cooperative manner, although they do so taking into account the actions of all other firms. Therefore, we assume firms make decisions to maximize profit in the sense of Nash equilibrium. The argument of the profit function that each firm maximizes over depends on the mode of competition, but in all cases the profit function is given by

$$
\Pi_{i}=q_{i} \cdot\left(p_{i}-s_{i}\right) .
$$

Remark 3.1. If we started with firms having individual $\alpha_{i}$ 's in Equation (1), then this could be reduced to the identical $\alpha$ case by absorbing each $\alpha_{i}$ into the cost $s_{i}$ in Equation (7). However, we do not treat the case of individual $\beta$ 's or $\gamma$ 's here.

We suppose that the $N$ firms are ordered by cost such that, possibly after a suitable relabeling, $0<s_{1} \leq s_{2} \leq \cdots \leq s_{N}$. Further, we assume that $s_{N}<\alpha$, that is every firm's cost is lower than the maximum possible market price. In the next two subsections, we present the Nash equilibria results for the Cournot and Bertrand games for markets with differentiated goods. The results for the homogeneous goods markets under Cournot and Bertrand competition are given in Appendix A.

\subsection{Differentiated Cournot Competition}

Throughout this section, we roughly follow [13] wherein the results of the homogeneous Cournot game with general price functions are given. Although here we are dealing with the differentiated Cournot model, the method for constructing the Nash equilibrium is very similar for the case of linear inverse demands. We shall present here the full details of the construction in the differentiated goods setting.

The profit functions of the firms are given in Equation (7), and here each firm $i$ maximizes over $q_{i}$ with price given as the function of $q$ in System (2). If all the equilibrium quantities are strictly positive, then the first-order conditions give the best-response function for a given firm:

$$
q_{i}^{B R}=\frac{1}{2 \beta}\left(\alpha-\gamma \sum_{j \neq i} q_{j}-s_{i}\right), \quad i=1, \ldots, N .
$$

We can sum these equations over $i$ to obtain

$$
0=-2 \beta Q+N \alpha-\gamma(N-1) Q-S^{(N)},
$$

where we let $S^{(n)}=\sum_{j=1}^{n} s_{j}$, the sum of the costs of the first $n$ firms. We define the candidate equilibrium total production $Q^{\star, N}$, as the solution to the scalar equation $f_{N}(Q)=S^{(N)}$, where

$$
f_{N}(Q)=N \alpha-(2 \beta+(N-1) \gamma) Q, \quad Q>0 .
$$


Let us define the following effective market price function: $\bar{P}(Q)=\alpha-\gamma Q$. This is not an actual price in the market, but it is a useful mathematical tool. We use the description effective as we shall see that this price serves the purpose of the single price in the market that all costs must be compared to. With this market price function, we can rearrange Equation (8) to obtain a candidate Nash equilibrium

$$
q_{i}^{\star, N}=\frac{\bar{P}\left(Q^{\star, N}\right)-s_{i}}{2 \beta-\gamma}, \quad i=1, \ldots, N .
$$

Utilizing Equations (9) and (10), we can express the candidate equilibrium as

$$
q_{i}^{\star, N}=\left(\frac{\bar{P}\left(Q^{\star, N}\right)-s_{i}}{\sum_{j=1}^{N}\left(\bar{P}\left(Q^{\star, N}\right)-s_{j}\right)}\right) Q^{\star, N} .
$$

Equation (11) is convenient for expressing the candidate equilibrium quantities as it has the interpretation that once the total equilibrium quantity $Q^{\star, N}$ is determined, each player produces the fraction which is the deviation of his cost $s_{i}$ from the effective market price $\bar{P}\left(Q^{\star, N}\right)$ relative to the total deviation of all players' costs from that price.

However, it may occur that $q_{i}^{\star, N}<0$ for some $i$, which is not an admissible solution and we must thus consider equilibria with less than $N$ firms. For $1 \leq n \leq N$, we define

$$
f_{n}(Q)=n \alpha-(2 \beta+(n-1) \gamma) Q, \quad Q>0 .
$$

Observe that $f_{n}(Q)$ is strictly decreasing in $Q$ for all $n$. For a fixed $n \in\{1, \ldots, N\}$, there is a unique $Q^{\star, n}$ such that $f_{n}\left(Q^{\star, n}\right)=S^{(n)}$, which is given by

$$
Q^{\star, n}=\frac{n \alpha-S^{(n)}}{2 \beta+(n-1) \gamma} .
$$

For each $n$, we have the following $n$-player candidate equilibrium:

$$
q_{i}^{\star, n}=\left\{\begin{array}{cl}
\frac{\bar{P}\left(Q^{\star, n}\right)-s_{i}}{2 \beta-\gamma} & \text { for } 1 \leq i \leq n, \\
0 & \text { for } n+1 \leq i \leq N,
\end{array}\right.
$$

where $Q^{\star, n}$ is given in Equation (13). We denote the corresponding prices by $p_{i}^{\star, n}$ using System (2). In order to determine which of the candidate equilibria is the true equilibrium of the game, we first compute, for a given firm, the net revenue per unit as

$$
p_{i}^{\star, n}-s_{i}=\frac{\beta}{2 \beta-\gamma}\left(\bar{P}\left(Q^{\star, n}\right)-s_{i}\right) .
$$

The following lemma then follows directly from Equations (14) and (15).

Lemma 3.1. $q_{i}^{\star, n}<0$ if and only if $p_{i}^{\star, n}<s_{i}$.

A given candidate equilibrium can fail to be a Nash equilibrium of the game if

(i) $q_{i}^{\star, n}<0$ for some $1 \leq i \leq n$, or

(ii) $s_{i}<p_{i}\left(q_{1}^{\star, n}, \ldots, q_{n}^{\star, n}, 0, \ldots, 0\right)$ for some $n+1 \leq i \leq N$. 
Case (i) holds if and only if $s_{i}>\bar{P}\left(Q^{\star, n}\right)$. So the effective market price is too low for this player and they would be better off not producing at all. Thus, we should look for a Nash equilibrium with a smaller number of active players. We provide the following lemma before discussing the second case.

Lemma 3.2. $s_{n}<p_{n}\left(q_{1}^{\star, n-1}, \ldots, q_{n-1}^{\star, n-1}, 0, \ldots, 0\right)$ if and only if $s_{n}<\bar{P}\left(Q^{\star, n-1}\right)$. In other words, Firm $n$ wishes to participate in the $(n-1)$-firm equilibrium if and only if $s_{n}<\bar{P}\left(Q^{\star, n-1}\right)$.

Proof. The result follows from the equivalence

$$
p_{n}\left(q_{1}^{\star, n-1}, \ldots, q_{n-1}^{\star, n-1}, 0, \ldots, 0\right)=\alpha-\beta(0)-\gamma \sum_{j=1}^{n-1} q_{j}^{\star, n-1}=\alpha-\gamma Q^{\star, n-1}=\bar{P}\left(Q^{\star, n-1}\right) .
$$

Case (ii) means that some firm $i \in\{n+1, \ldots, N\}$ could produce a strictly positive quantity and would receive a price strictly above cost from the market and therefore make a positive profit. Lemma 3.2 shows that this case occurs if and only if $s_{i}<\bar{P}\left(Q^{\star, n}\right)$. In this case we should look for a Nash equilibrium with a larger number of active players.

The procedure for determining the equilibrium is to start with the one firm equilibrium that consists of the lowest cost firm producing as a monopoly. We then consider whether Firm 2 wishes to participate. That is, we check if Case (ii) holds with $i=2$. If so, we ask if both wish to participate in the two-player candidate equilibrium. We continue in this manner until we run out of firms to add or additional firms do not wish to enter. This construction has similarities to the equilibrium constructions found in [4] and [11], in that there is a cutoff point below which firms produce and above which firms are costed out. We must, however, take care that this procedure terminates and that this is sufficient to determine a unique Nash equilibrium. We establish in the following lemma the necessary inductive step.

Lemma 3.3. Fix some $n<N$. We have $n$ and $(n+1)$-player candidate equilibria with total production quantities $Q^{\star, n}$ and $Q^{\star, n+1}$, respectively, and the individual firm production quantities given by Equation (14) with the corresponding $Q^{\star}$. Then, Firm $n+1$ will want to be active in the $n$-firm equilibrium if and only if they want to be active in the $(n+1)$-firm equilibrium.

Proof. From Lemma 3.2 Firm $n+1$ wants to be active in the $n$-firm equilibrium if and only if $s_{n+1}<\bar{P}\left(Q^{\star, n}\right)$. Recall the definition of $Q^{\star, n}$ as the unique $Q$ which satisfies $f_{n}\left(Q^{\star, n}\right)=S^{(n)}$, where the $f_{n}$ are defined in Equation (12) and are strictly decreasing for $Q>0$. One can show for $1 \leq n<N$ that $f_{n+1}\left(Q^{\star, n}\right)=S^{(n)}+\bar{P}\left(Q^{\star, n}\right)$, and therefore

$$
f_{n+1}\left(Q^{\star, n+1}\right)-f_{n+1}\left(Q^{\star, n}\right)=s_{n+1}-\bar{P}\left(Q^{\star, n}\right) .
$$

In a similar manner, one can show $f_{n}\left(Q^{\star, n+1}\right)=S^{(n+1)}-\bar{P}\left(Q^{\star, n+1}\right)$, and hence

$$
f_{n}\left(Q^{\star, n+1}\right)-f_{n}\left(Q^{\star, n}\right)=s_{n+1}-\bar{P}\left(Q^{\star, n+1}\right) .
$$


We then find

$$
\begin{aligned}
& s_{n+1}<\bar{P}\left(Q^{\star, n}\right) \Longleftrightarrow f_{n+1}\left(Q^{\star, n+1}\right)<f_{n+1}\left(Q^{\star, n}\right) \quad \text { from Equation (16), } \\
& \Longleftrightarrow Q^{\star, n+1}>Q^{\star, n} \quad \text { as } f_{n+1} \text { is decreasing, } \\
& \Longleftrightarrow f_{n}\left(Q^{\star, n+1}\right) \quad<f_{n}\left(Q^{\star, n}\right) \quad \text { as } f_{n} \text { is decreasing, } \\
& \Longleftrightarrow s_{n+1} \quad<\bar{P}\left(Q^{\star, n+1}\right) \quad \text { from Equation (17), }
\end{aligned}
$$

from which the conclusion follows.

Corollary 3.1. The total candidate equilibrium quantity is strictly increasing from $n$ firms to $(n+1)$ firms if and only if Firm $(n+1)$ is active in the $(n+1)$-firm candidate equilibrium.

Proof. The corollary states: $Q^{\star, n+1}>Q^{\star, n}$ if and only if $q_{n+1}^{\star, n+1}>0$. The conclusion follows from the proof of Lemma 3.3 as we have $s_{n+1}<\bar{P}\left(Q^{\star, n}\right) \Longleftrightarrow Q^{\star, n+1}>Q^{\star, n}$, and the lemma itself gives that this is equivalent to Firm $n+1$ being active in the $(n+1)$-firm candidate equilibrium.

Proposition 3.1. There exists a unique Nash equilibrium to the differentiated Cournot game. The unique equilibrium quantities are given by

$$
q^{\star}=\left(q_{1}^{\star, n^{\star}}, \ldots, q_{n^{\star}}^{\star n^{\star}}, 0, \ldots, 0\right),
$$

where $q_{i}^{\star, n}$ is given in Equation (14) and $n^{\star}$ is the number of active firms in equilibrium which is given by

$$
n^{\star}=\min \left\{n \in\{1, \ldots, N\}: Q^{\star, n}=\bar{Q}^{\star}\right\},
$$

with $\bar{Q}^{\star}=\max \left\{Q^{\star, n}: 1 \leq n \leq N\right\}$.

Proof. We know by assumption that Firm 1 will participate in the one-firm candidate equilibrium as $s_{1}<\alpha$. Hence $Q^{\star, 1}>0$. Suppose that for some $n<N$ we have a candidate Nash equilibrium in which the first $n$ firms are active. If $s_{n+1}<\bar{P}\left(Q^{\star, n}\right)$, or equivalently if $Q^{\star, n+1}>Q^{\star, n}$, then from Lemma 3.3, Firm $n+1$ wishes to enter, and by the same lemma, they will participate in the $(n+1)$-firm equilibrium. Furthermore, as all the other $n$ firms have costs lower than or equal to Firm $n+1$, they will also be active in the $(n+1)$-firm equilibrium. Therefore, every candidate equilibrium with $n$ or fewer players cannot be a true equilibrium due to entry. We can proceed adding players until either no further players wish to enter or there are no further players. We have uniqueness by construction.

The explicit characterization of $n^{\star}$, the number of active firms in equilibrium, comes from the fact that $Q^{\star, n}$ is strictly increasing in $n$ as firms enter. At some point, we may have $Q^{\star, n}=Q^{\star,(n-1)}$ if a firm is exactly indifferent between entering and not entering, i.e. $q_{n}^{\star, n}=0$. Such a firm is not considered active in the equilibrium. It may stay flat for a range of $n$ if there is a range of firms with identical costs all of which are indifferent between entering and not entering. Then, the sequence will be decreasing. Thus, $n^{\star}$ will be given by the first $n$ where $Q^{\star, n}$ attains its maximum $\bar{Q}^{\star}$. In other words, $n^{\star}=\min \left\{n \in\{1, \ldots, N\}: Q^{\star, n}=\bar{Q}^{\star}\right\}$.

We illustrate the behavior of $Q^{\star, n}$ in Figure 1. In this example, the number of active firms in equilibrium, $n^{\star}$, is 5 , while $N=9$. This figure was generated with $\alpha=1, \beta=0.5, \gamma=0.2$. We also set the vector of costs to $s=(0.25,0.27,0.35,0.6,0.6,0.67 \overline{4}, 0.67 \overline{4}, 0.72,0.85)$. Firms 4 and 5 have equal cost and as $Q^{\star, 5}>Q^{\star, 4}$, we know that both are active in equilibrium. We also note that 
Firms 6 and 7 have equal cost, greater than the cost of Firm 5, and are exactly indifferent between entering and not entering. We see this as $Q^{\star, 5}=Q^{\star, 6}=Q^{\star, 7}$. The shape of the curve appears to always be concave, although we have not explored whether this holds in general. The main important feature to note is that the curve is strictly increasing up to $n^{\star}$ and flat or decreasing after $n^{\star}$.

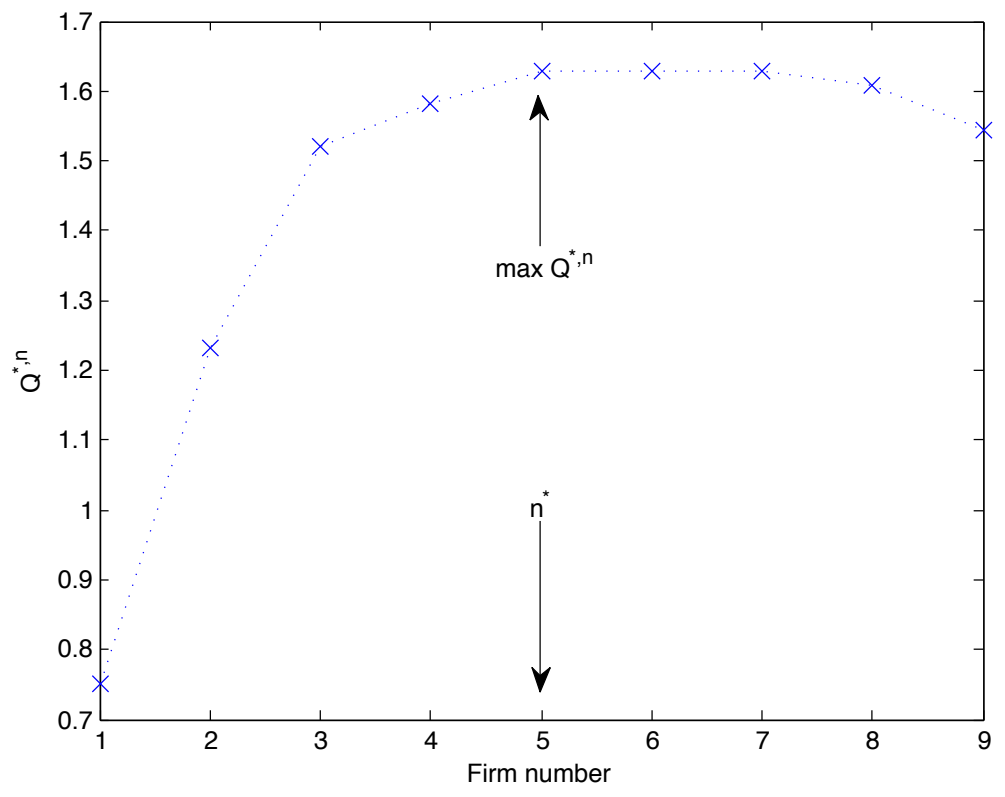

Figure 1: Illustrative behavior of $Q^{\star, n}$ and the number of active firms in equilibrium.

\subsection{Differentiated Bertrand Competition}

The profit function that the firms seek to maximize is again given in Equation (7), where the firms now choose $p_{i}$ and quantities are given by $q_{i}(p)$ in System (3). In addition, here we assume that if a firm receives zero demand in equilibrium, they set price equal to cost. The method of solution for this model is quite similar on the surface to the differentiated Cournot model. We can solve for the firms' best-response functions and obtain candidate Nash equilibria from their intersection.

The reason for considering different candidate equilibria is that, again, in the game with heterogeneous costs, the candidate equilibrium with all $N$ firms may result in negative demands for some firms. We must thus consider subgames which, for $n=1, \ldots, N$, involve only the first $n$ players. Let $p^{\star, n}=\left(p_{1}^{\star, n}, \ldots, p_{n}^{\star, n}, s_{n+1}, \ldots, s_{N}\right)$, where the first $n$ components solve the Nash equilibrium problem with profit functions as described above, and with $N$ replaced by $n$ in the coefficients of the demand functions $q_{i}(p)$ given in System (3). These are given explicitly in Equation (18).

Let $p^{\star}$ denote the vector of prices in equilibrium. It is shown in [19] that the Nash Equilibrium of the Bertrand game will be one of three types:

$\langle\mathrm{I}\rangle$ All $N$ firms price above cost. In this case, $p_{i}^{\star}>s_{i}$ for all $i=1, \ldots, N$, and the Nash equilibrium is simply the $N$-player interior Nash equilibrium given by $p^{\star}=\left(p_{1}^{\star, N}, \ldots, p_{N}^{\star, N}\right)$, where the $p_{i}^{\star, N}$ are as defined above. 
$\langle$ II $\rangle$ For some $0 \leq n<N$, firms $1, \ldots, n$ price strictly above cost and the remaining firms set price equal to cost. In other words, $p_{i}^{\star}>s_{i}$ for $i=1, \ldots, n$, and $p_{j}=s_{j}$ for $j+1, \ldots, N$. The first $n$ firms play the interior $n$-player sub-game equilibrium as if firms $n+1, \ldots, N$ did not exist. These firms are completely ignorable because their costs are too high. The high cost firms receive zero demand and have prices fixed at cost by our definition of Nash equilibrium. The Nash equilibrium is $p^{\star}=\left(p_{1}^{\star, n}, \ldots, p_{n}^{\star, n}, s_{n+1}, \ldots, s_{N}\right)$.

$\langle$ III $\rangle$ For some $(k, n)$ such that $0 \leq k<n \leq N$, firms $1, \ldots, k$ price strictly above cost (if $k=0$ then no firms price strictly above cost), and the remaining firms set price equal to cost. In other words, $p_{i}^{\star}>s_{i}$ for $i=1, \ldots, k$ and $p_{j}^{\star}=s_{j}$ for $j=k+1, \ldots, N$.

Type $\langle\mathrm{III}\rangle$ differs from Type $\langle\mathrm{II}\rangle$ in that firms $k+1, \ldots, n$ are not ignorable: their presence is felt in the pricing decisions of firms $1, \ldots, k$, and we say that firms $k+1, \ldots, n$ are on the boundary. Similar to Type $\langle\mathrm{II}\rangle$, firms $n+1, \ldots, N$ are still completely ignorable. If firms $1, \ldots, k$ ignore firms $k+1, \ldots, n$ and set $k$-player Nash equilibrium prices, then the latter firms will set a price above cost which contradicts their being ignored. On the other hand, if we consider firms $k+1, \ldots, n$ to be fully active in equilibrium, then their optimal prices in the $n$-player Nash candidate equilibrium would not be above their costs. Therefore, there is the Type $\langle\mathrm{III}\rangle$ equilibrium where firms $k+1, \ldots, n$ are not active in the equilibrium, but firms $1, \ldots, k$ set prices that take the presence of the former firms into consideration.

Only an equilibrium of Type $\langle\mathrm{I}\rangle$ or Type $\langle\mathrm{II}\rangle$ is possible in the Cournot game. There we had Lemma 3.2 which resolved the fact that a firm whose quantity is negative in a candidate equilibrium does not wish to enter the game with a positive quantity if the remaining firms ignore this firm and play the candidate Nash equilibrium with one less player. However, in the Bertrand game, such a result does not hold. The unique Nash equilibrium is given explicitly in the following.

Proposition 3.2 (Prop 2.6 in [19]). There exists a unique equilibrium to the Bertrand game with linear demand. The type $\langle I\rangle$ and $\langle I I\rangle$ candidate solutions are given by

$$
p_{i}^{\star, n}=\frac{1}{\left(2 b_{n}+c_{n}\right)}\left[a_{n}+c_{n} \frac{n a_{n}+b_{n} \sum_{m=1}^{n} s_{m}}{\left(2 b_{n}-(n-1) c_{n}\right)}+b_{n} s_{i}\right] .
$$

The type $\langle I I I\rangle$ candidate solutions are given by

$$
\begin{aligned}
p_{i}^{b, n+1, n+1-k}= & \frac{1}{\left(2 b_{n+1}+c_{n+1}\right)}\left[\left(a_{n+1}+c_{n+1} \sum_{m=k+1}^{n+1} s_{m}\right)\right. \\
& \left.+c_{n+1}\left(\frac{n\left(a_{n+1}+c_{n+1} \sum_{m=k+1}^{n+1} s_{m}\right)+b_{n+1} \sum_{m=1}^{k} s_{m}}{2 b_{n+1}-(k-1) c_{n+1}}\right)+b_{n+1} s_{i}\right],
\end{aligned}
$$

where the superscript $(b, n+1, n+1-k)$ stands for boundary, $(n+1)$ firms entering into the demand function, and $n+1-k$ firms on the boundary, i.e. not ignorable. The Nash equilibrium is constructed as follows:

- If $s_{1}>\frac{a_{1}}{b_{1}}$, then $p^{\star}=\left(s_{1}, \ldots, s_{N}\right)$. (Type $\langle I I\rangle$ with $\left.n=N\right)$.

- Else, find $n<N$ such that $p_{i}^{\star, n}>s_{i}, \forall i=1, \ldots, n$, and $p_{n+1}^{\star, n+1} \leq s_{n+1}$. 
- If $s_{n+1} \geq b_{n+1}^{-1}\left(a_{n+1}+c_{n+1} \sum_{i=1}^{n} p_{i}^{\star, n}\right)$, then $p^{\star}=\left(p_{1}^{\star, n}, \ldots, p_{n}^{\star, n}, s_{n+1}, \ldots, s_{N}\right)$, (Type $\langle$ II $\rangle$ with $n<N)$,

- Else,

○ if $p_{n}^{b, n+1,1}>s_{n}$, then $p^{\star}=\left(p_{1}^{b, n+1,1}, \ldots, p_{n}^{b, n+1,1}, s_{n+1}, \ldots, s_{N}\right)$, (Type $\left.\langle I I I\rangle\right)$,

- else, find $k<n$ such that

$$
\begin{gathered}
p_{i}^{b, n+1, n+1-k}>s_{i} \text { for all } i=1, \ldots, k, \quad \text { and } p_{k+1}^{b, n+1, n+1-(k+1)}<s_{k+1} . \\
\text { Then } p^{\star}=\left(p_{1}^{b, n+1, n+1-k}, \ldots, p_{k}^{b, n+1, n+1-k}, s_{k+1}, \ldots, s_{N}\right) \cdot(\text { Type }\langle\text { III }\rangle) .
\end{gathered}
$$

The Nash equilibria for the homogeneous Cournot and Bertrand games are given in the Appendix.

\section{How many firms are active?}

In this section we consider the question of whether a firm is active or inactive in equilibrium. The main result of this section is an ordering of the number of active firms in equilibrium across the four different types of games. Following the analytic result, we provide numerical examples to demonstrate the various prospective outcomes.

\subsection{The General Case: N-Firm Games}

Fix the total number of firms $N$, the parameters $\alpha, \beta$, and $\gamma$, and the vector of costs $s=\left(s_{1}, \ldots, s_{N}\right)$ such that $0<s_{1} \leq s_{2} \leq \cdots \leq s_{N}<\alpha$.

We shall denote by $n_{B h}^{\star}, n_{B d}^{\star}, n_{C h}^{\star}, n_{C d}^{\star}$, the number of active firms in equilibrium in the homogeneous Bertrand game, differentiated Bertrand game, homogeneous Cournot game and differentiated Cournot game, respectively. The purpose of this section is to show specifically how these numbers relate to one another. This will be shown in Theorem 4.1, but first we establish three helpful lemmas. In the process we shall need the quantity $\theta_{n}=(\beta-\gamma)\left(\frac{2 \beta+(n-1) \gamma}{\beta+(n-2) \gamma}\right)$.

Recall the fact that in both Cournot type games, a firm is active in equilibrium, if and only if the total equilibrium supply strictly increases when this firm enters the market. In the homogeneous Cournot game, this amounts to the fact that Firm $n$ is active if and only if $Q_{H}^{\star, n}>Q_{H}^{\star, n-1}$. In the differentiated Cournot game, the condition is $Q^{\star, n}>Q^{\star, n-1}$ if and only if Firm $n$ is active in equilibrium. We characterize these conditions in terms of the cost of the firms in the following lemma.

Lemma 4.1. In the homogeneous Cournot game, we have

$$
Q_{H}^{\star, n}>Q_{H}^{\star, n-1} \Longleftrightarrow s_{n}<\frac{\alpha+S^{(n-1)}}{n} .
$$

In the differentiated Cournot game, we have

$$
Q^{\star, n}>Q^{\star, n-1} \quad \Longleftrightarrow \quad s_{n}<\frac{\alpha(2 \beta-\gamma)+\gamma S^{(n-1)}}{(2 \beta-\gamma)+(n-1) \gamma} .
$$


Proof. For the homogeneous case, the definition of $Q_{H}^{\star, n}$ is given in Equation (58) in Appendix A. Then the result follows by writing down $Q_{H}^{\star, n}-Q_{H}^{\star, n-1}$ and rearranging. The differentiated case can be found in Corollary 3.1, where we note that $s_{n}<\bar{P}\left(Q^{\star, n}\right)$ is equivalent to the given condition.

Lemma 4.2. The following expression is increasing in $h$

$$
\frac{\alpha h+\gamma S^{(n-1)}}{h+(n-1) \gamma}
$$

provided the denominator is not zero.

Proof. Take a derivative with resect to $h$ to obtain

$$
\frac{\partial}{\partial h}\left[\frac{\alpha h+\gamma S^{(n-1)}}{h+(n-1) \gamma}\right]=\frac{1}{(h+(n-1) \gamma)^{2}}\left(\alpha \gamma(n-1)-\gamma S^{(n-1)}\right) .
$$

As $\gamma$ is strictly positive, we find that this expression is strictly positive provided $(n-1) \alpha>S^{(n-1)}$. This is clearly true based on the assumption that $s_{i}<\alpha$ for all $i$.

Lemma 4.3. $(2 \beta-\gamma)>\theta_{n}$ for all $n>1$.

Proof.

$$
\begin{aligned}
2 \beta-\gamma-\theta_{n} & =2 \beta-\gamma-(\beta-\gamma)\left(\frac{2 \beta+(n-1) \gamma}{\beta+(n-2) \gamma}\right) \\
& =\gamma\left(\frac{(n-2) \beta+\gamma}{\beta+(n-2) \gamma}\right) .
\end{aligned}
$$

Obviously the Expression (19) is positive for $n>1$.

Theorem 4.1. $n_{B h}^{\star} \leq n_{C h}^{\star} \leq n_{C d}^{\star}$. Further, $n_{B d}^{\star} \leq n_{C d}^{\star}$. Finally, $n_{C h}^{\star} \geq n_{B d}^{\star}$ if $\theta_{n_{C h}^{\star}}<\gamma$.

Proof. $\underline{n_{B h}^{\star} \leq n_{C h}^{\star}}$

First, we note that $n_{B h}^{\star}=\#\left\{k: s_{k}=\min _{i} s_{i}\right\}$. Thus, this is equal to one unless we have for some $j, 0<s_{1}=\cdots=s_{j}<s_{j+1} \leq s_{j+2} \leq \cdots \leq s_{N}$. In such a case, we would have $n_{B h}^{\star}=j$. Our assumption $s_{1}<\alpha$ implies that $Q_{H}^{\star, 1}>0$. Furthermore, with the above specification for the costs, simple algebra shows

$$
Q_{H}^{\star, j}=2\left(\frac{j}{j+1}\right) Q_{H}^{\star, 1}
$$

Moreover, $2\left(\frac{j}{j+1}\right)>1$ if and only if $j>1$. Hence, $Q_{H}^{\star, j}>Q_{H}^{\star, 1}$ for all $j>1$. Therefore at least all firms with cost equal to the lowest cost firm are active in the homogeneous Cournot equilibrium. In this case, this means $n_{C h}^{\star} \geq j$. Therefore, $n_{B h}^{\star} \leq n_{C h}^{\star}$.

$\underline{n_{C h}^{\star}} \leq n_{C d}^{\star}:$

Lemma 4.1 specifies that firm $n$ participates in the homogeneous Cournot equilibrium if

$$
s_{n}<\frac{\alpha+S^{(n-1)}}{n}=\frac{\alpha \gamma+\gamma S^{(n-1)}}{\gamma+(n-1) \gamma},
$$


and this firm participates in the differentiated Cournot equilibrium if

$$
s_{n}<\frac{\alpha(2 \beta-\gamma)+S^{(n-1)}}{2 \beta+(n-2) \gamma} .
$$

Using Lemma 4.2, we have

$$
\frac{\alpha(2 \beta-\gamma)+S^{(n-1)}}{(2 \beta-\gamma)+(n-1) \gamma}>\frac{\alpha \gamma+\gamma S^{(n-1)}}{\gamma+(n-1) \gamma},
$$

because $(2 \beta-\gamma)>\gamma$. Hence, if Firm $n$ is active in the homogeneous Cournot equilibrium, i.e. if their cost is low enough, then the firm is also active in the differentiated Cournot equilibrium by Expression (21). Therefore, $n_{C h}^{\star} \leq n_{C d}^{\star}$.

$\underline{n_{B d}^{\star} \leq n_{C d}^{\star}}$

The differentiated Bertrand equilibrium can be of three types. In Type $\langle\mathrm{I}\rangle$, we have that all firms price above cost according to their interior candidate equilibrium prices $p_{i}^{\star, N}$. The condition $p_{N}^{\star, N}>s_{N}$ is necessary and sufficient for this equilibrium to hold. This condition on $s_{N}$ is equivalent to

$$
s_{N}<\frac{\alpha \theta_{N}+\gamma S^{(N-1)}}{\theta_{N}+(N-1) \gamma}
$$

Using Lemmas 4.2 and 4.3, we find that

$$
\frac{\alpha \theta_{N}+\gamma S^{(N-1)}}{\theta_{N}+(N-1) \gamma}<\frac{\alpha(2 \beta-\gamma)+S^{(N-1)}}{(2 \beta-\gamma)+(N-1) \gamma} .
$$

This implies that if there is a Type $\langle\mathrm{I}\rangle$ equilibrium in the differentiated Bertrand game, i.e. $n_{B d}^{\star}=$ $N$, then we also have $n_{C d}^{\star}=N$.

If we a priori know that we have an equilibrium of Type $\langle\mathrm{II}\rangle$, then we know that Firm $n$ is active in the differentiated Bertrand equilibrium if

$$
s_{n}<\frac{\alpha \theta_{n}+\gamma S^{(n-1)}}{\theta_{n}+(n-1) \gamma},
$$

and the firm is active in the differentiated Cournot equilibrium if

$$
s_{n}<\frac{\alpha(2 \beta-\gamma)+S^{(n-1)}}{(2 \beta-\gamma)+(n-1) \gamma}
$$

Again using Lemmas 4.2 and 4.3, we find the same inequality as in Expression (22) with $N$ replaced by $n$. Hence, a firm being active in the Type $\langle\mathrm{II}\rangle$ differentiated Bertand equilibrium implies the firm is active in the differentiated Cournot equilibrium. So for a Type $\langle$ II $\rangle$ equilibrium, we have $n_{B d}^{\star} \leq n_{C d}^{\star}$.

Finally, the situation of a Type $\langle$ III $\rangle$ equilibrium could arise. However, this serves only to decrease the number of active firms in the market from the maximum number of firms that could be sustained in a Type $\langle\mathrm{II}\rangle$ equilibrium. That is, a Type $\langle\mathrm{III}\rangle$ equilibrium starts with a Type $\langle\mathrm{II}\rangle$ equilibrium and then possibly removes some firms from being active. Therefore, in all cases we have $n_{B d}^{\star} \leq n_{C d}^{\star}$.

$\underline{n_{C h}^{\star} \text { vs } n_{B d}^{\star}}$ 
Let us ignore for now the case of a Type $\langle\mathrm{III}\rangle$ equilibrium in the differentiated Bertrand game. We have established the conditions on $s_{n}$ for a firm to be active in the two types of equilibria. First, in the homogeneous Cournot case we have the condition given in Expression (20). Second, in a Type $\langle\mathrm{I}\rangle$ or Type $\langle\mathrm{II}\rangle$ equilibrium in the differentiated Bertrand game, we have the condition given in Expression (23). We find by Lemma 4.2 that Firm $n$ being active in the differentiated Bertrand game implies the firm is active in the homogeneous Cournot game if $\theta_{n_{C h}^{\star}}<\gamma$. Therefore, this implies $n_{B d}^{\star} \leq n_{C h}^{\star}$. This result continues to hold for a Type $\langle$ III $\rangle$ equilibrium as again such an equilibrium only serves to reduce $n_{B d}^{\star}$.

Remark 4.1. If $\theta_{n_{B d}^{\star}}>\gamma$ then the relative size of the conditions on cost reverses and therefore one expects $n_{C h}^{\star} \leq n_{B d}^{\star}$ to hold. However, this does not account for the possibility of a Type $\langle$ III $\rangle$ equilibrium. The number of active firms in a Type $\langle$ III $\rangle$ equilibrium is highly sensitive to the costs and it is quite difficult to ascertain what occurs in this case. However, Type $\langle$ III $\rangle$ equilibria are rare in the sense that they only occur for very special specifications of the costs. Therefore, in most situations, one should have $n_{C h}^{\star} \leq n_{B d}^{\star}$ if $\theta_{n_{B d}^{\star}}>\gamma$.

Remark 4.2. The condition $\theta_{n}>\gamma$ essentially boils down to $\gamma$ being small enough relative to $\beta$. In fact, one can show that $\theta_{n}-\gamma=(2 \beta+n \gamma)(\beta-2 \gamma)+3 \gamma^{2}$. Thus, a sufficient condition for this to be positive is $\beta>2 \gamma$. Moreover, what happens as $\gamma$ approaches $\beta$ is that the homogeneous Cournot and the differentiated Cournot approach one another, and similarly with the two Bertrand models. However, as the homogeneous Bertrand model always has only one active firm in the market (when there is a unique low cost firm) and the differentiated Cournot model has the most active, we find that the differentiated Bertrand and homogeneous Cournot have to cross over at some point to account for this lack of differentiation among the goods. Thus, informally, one can claim that for sufficiently differentiated products we have: $n_{B h}^{\star} \leq n_{C h}^{\star} \leq n_{B d}^{\star} \leq n_{C d}^{\star}$.

Remark 4.3. If we let $\gamma=0$, then the differentiated Bertrand and Cournot models coincide in terms of the number of active firms. This can be seen from Expressions (23) and (24), as setting $\gamma=0$ causes $\theta_{n}=2 \beta$ and the conditions on cost for a firm to be active in equilibrium in the two cases then coincide. Also, based on the assumption that $s_{i}<\alpha$ for all $i$, we will have all firms active in the equilibrium that results with $\gamma=0$. This is all fairly obvious but at least worthwhile to note as the behavior of the number of active firms in equilibrium depends highly on $\gamma$ and there is no difference between differentiated Bertrand and Cournot when goods are independent.

\subsection{Numerical Examples}

Fix $s=(0.25,0.27,0.35,0.4,0.5), \alpha=1$ and $\beta=0.5$. We then consider the addition of another firm to the market with cost $s_{6}$ varying from 0.001 to 0.999 . We consider three different possible values of $\gamma$, a low, base and high case. The low and high cases for the four different oligopoly models are displayed in Figure 2 (a) and (b). The results for the base case of $\gamma=0.3$ are displayed in Figure 3.

In the high case, we see that increasing $\gamma$ this much towards $\beta$ causes the number of active firms in the differentiated Bertrand market to be less than that of the homogeneous Cournot market. We also see that the homogeneous and differentiated Cournot markets have similar numbers of active firms, coinciding over a large region of the cost of the additional firm, contrary to the result in Figure 3 where they are completely separated over the whole space of costs. 


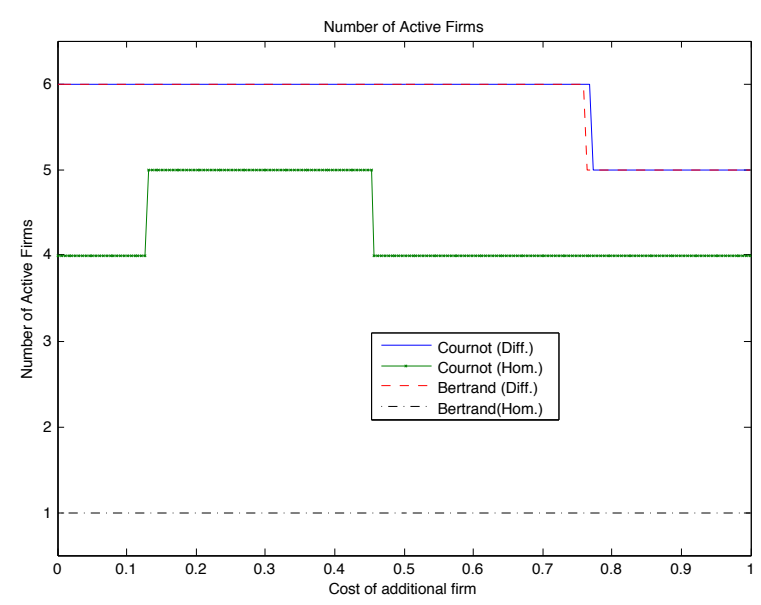

(a) Low, $\gamma=0.1$

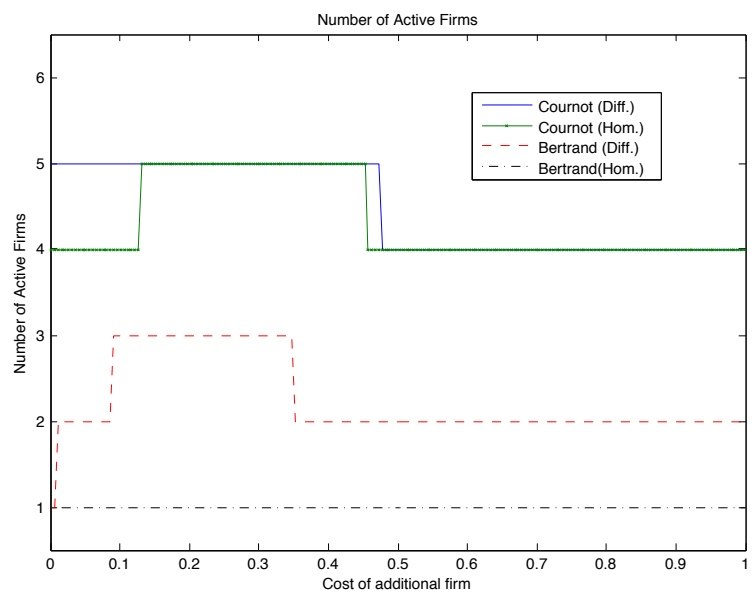

(b) High, $\gamma=0.45$

Figure 2: Number of Active Firms - 6 firm example

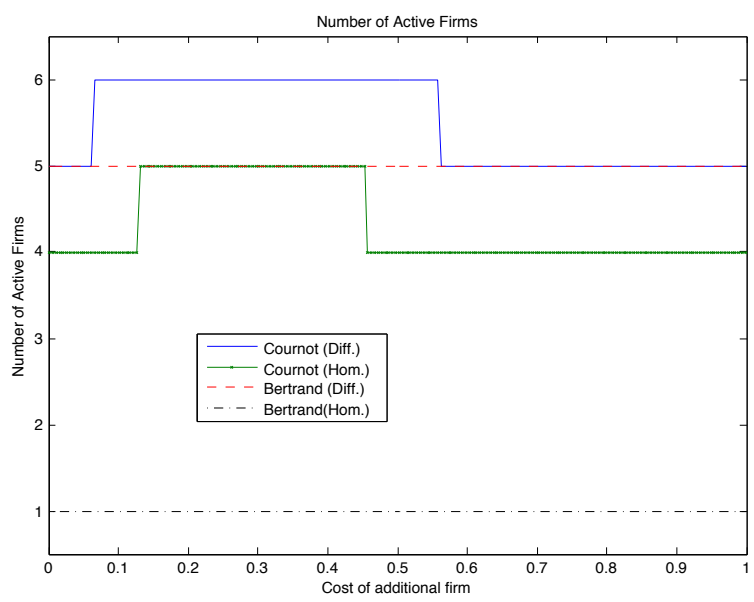

Figure 3: Number of Active Firms - 6 firm example, $\gamma=0.3$

In the low case representing the other extreme point of view where we move $\gamma$ towards zero, we see that the number of firms in the differentiated Bertrand and Cournot models (the top two lines in the figure which are on top of one another except between 0.7 and 0.8) are almost identical, with Cournot having more firms over a small region of the space of costs. We also see that the homogeneous Cournot is completely separated from the three other models of oligopoly.

Another point of view from which we can study the behavior of the effect of the degree of product differentiation on the market equilibrium is to plot the number of active firms in the four different market types versus the ratio $\gamma / \beta$. We fix the total possible number of firms at 40 , we set $\alpha=1$, and finally we fix $\beta=0.5$. We then vary $\gamma$ so that $\gamma / \beta$ ranges from 0 to 1 .

First, we set $s_{1}=0.1$ and $s_{40}=0.99$, and specify the costs of the intermediate firms to be linearly increasing from $s_{1}$ to $s_{40}$ so that the difference between the costs of adjacent firms is constant. We display this in Figure 4 (a).

Next, we put firms into 5 groups so that there are 8 firms with equally spaced costs within each 
group, the costs varying from lowest to highest by 0.005 , then with a gap of 0.18 between groups. For example, the first group has $s_{1}=0.1, s_{2}=0.105, s_{3}=0.110, \ldots, s_{8}=0.135$; then $s_{9}=0.315$. We continue in this manner until we get to $s_{40}=0.995$. We display the results with this cost structure in Figure 4 (b).

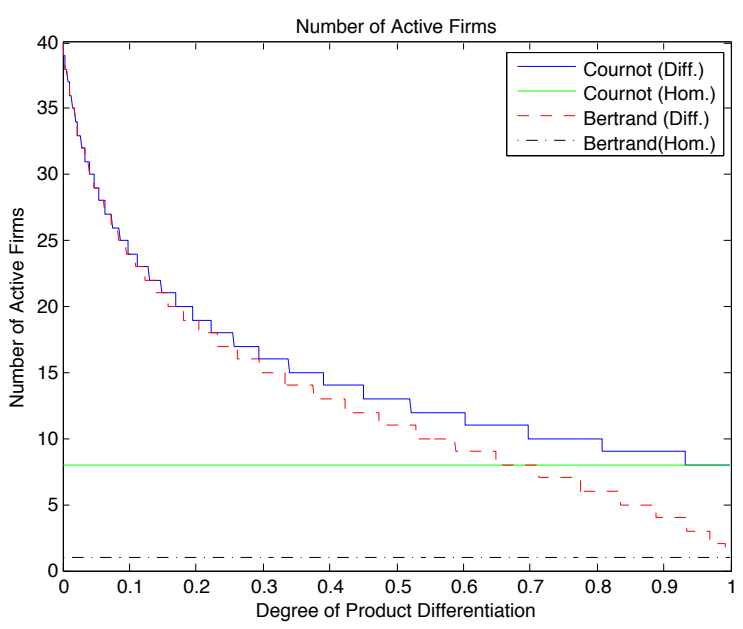

(a) Evenly spaced costs

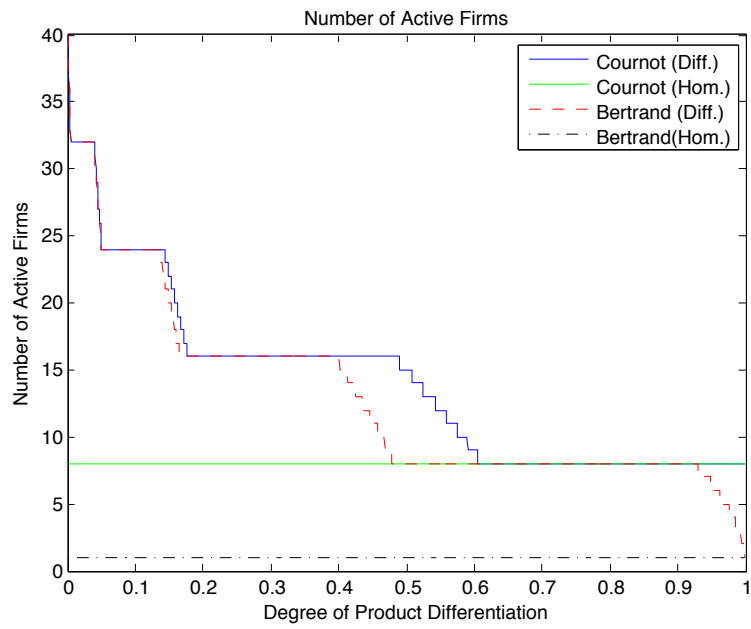

(b) Five different cost clusters

Figure 4: Number of Active Firms - 40 firm example

One can clearly see from both figures that the number of active firms in equilibrium is decreasing in the degree of product differentiation. That is, with $\gamma / \beta=0$, meaning independent goods, we have the maximum number of active firms, and with $\gamma / \beta=1$, meaning homogeneous goods, we have the minimum number of active firms. We can see the point where the differentiated Bertrand crosses over the homogeneous Cournot is at a high degree of product differentiation, thereby supporting our previous remark that for low values of $\gamma / \beta$ we expect $n_{B h}^{\star} \leq n_{C h}^{\star} \leq n_{B d}^{\star} \leq n_{C d}^{\star}$. Comparing the two figures, we see that with a nice linearly interpolated cost structure we get relatively smooth behavior of the number of active firm curves. However, with clustered costs, we can see that essentially each cluster is either wholly in or wholly out. This leads to the large flat regions of space with no change in structure, along with sudden large drops in the number of firms.

In a realistic dynamic situation as market conditions change, the number of active firms in equilibrium will vary over time. We illustrate this issue in a dynamic homogeneous Cournot model of energy production in the following section.

\section{A Dynamic Game With Asymmetric Costs}

Consider an energy market with one oil producer with exhaustible reserves and cost of production equal to zero. There are also $N-1$ producers of energy using alternative, costly but inexhaustible technologies such as solar or wind. These firms are identified and ordered by their unit costs of production, $s_{i}$, such that $0 \leq s_{1} \leq s_{2} \leq \cdots \leq s_{N-1}<1$, and we recall the notation $S^{(k)}=\sum_{j=1}^{k} s_{j}$. 
The oil producer (Player 0 ) has reserves $x(t)$ at time $t$, with the dynamics

$$
\frac{d x}{d t}=-\bar{q}_{0}(x(t)) \mathbb{1}_{\{x(t)>0\}}
$$

where $\bar{q}_{0}(x(t))$ is his production rate. When his reserves run out, he no longer participates in the market. Let $\bar{q}_{i}(x(t)), i=1, \ldots, N-1$ be the production rates of the alternative technology producers. Then, the price of energy in a homogeneous Cournot market is given by

$$
P(t)=1-\bar{q}_{0}(x(t))-\sum_{i=1}^{N-1} \bar{q}_{i}(x(t)),
$$

where in the notation of Section A.1 we have taken $\alpha=\beta=1$.

In the static Cournot oligopoly game, the Nash equilibrium $q_{i}^{\star}\left(s_{0}, s\right)$ as a function of the costs $s_{0}$ for Player 0 and the vector $s=\left(s_{1}, \ldots, s_{N-1}\right)$ for the others was given in Proposition A.1. We denote by $G_{i}\left(s_{0}, s\right), i=0, \ldots, N-1$, the static game equilibrium profit, and in fact $G_{i}\left(s_{0}, s\right)=\left(q_{i}^{\star}\left(s_{0}, s\right)\right)^{2}$.

The firms are maximizing lifetime profit, and once the oil producer runs out of oil, and goes out of business, the remaining firms with their inexhaustible resources repeatedly play a static game with profit flow $G_{i}(1, s)$ over an infinite time horizon. The first argument in $G_{i}$ is set to the choke price, 1, which means that Player 0 no longer participates, and it is an $N-1$ player game. Given initial oil reserves $x(0)=x$, the value functions, $v_{0}(x)$ for Player 0 and $w_{i}$ for Players $i=1, \ldots, N-1$ are

$$
\begin{aligned}
& v_{0}(x)=\sup _{\bar{q}_{0}} \int_{0}^{\infty} e^{-r t} \bar{q}_{0}(x(t))\left(1-\bar{q}_{0}(x(t))-\sum_{j=1}^{N-1} \bar{q}_{j}(x(t))\right) \mathbb{1}_{\{x(t)>0\}} d t, \\
& w_{i}(x)=\sup _{\bar{q}_{i}} \int_{0}^{\infty} e^{-r t} \bar{q}_{i}(x(t))\left(1-\bar{q}_{0}(x(t))-\sum_{j=1}^{N-1} \bar{q}_{j}(x(t))-s_{i}\right) \mathbb{1}_{\{x(t)>0\}} d t+\frac{1}{r} G_{i}(1, s),
\end{aligned}
$$

with $v_{0}(0)=0$ and $w_{i}(0)=G_{i}(1, s) / r$. The case $N=2$ was studied in [13] (except there, the oil producer became a renewable energy producer with the same cost $s_{1}$ upon exhaustion). Here we analyze the case of a genuine $N$-player oligopoly. See also [20] for the two-player game with exploration; and [12] for a related problem in the context of mean-field games.

We look for a Markov Perfect Nash equilibrium $\bar{q}_{i}^{\star}(x(t))$, for $i=0, \ldots, N-1$. The associated Hamilton-Jacobi equation for $v_{0}$ is given by

$$
r v_{0}=G_{0}\left(v_{0}^{\prime}, s\right), \quad v_{0}(0)=0 .
$$

Here $s_{0}=v_{0}^{\prime}(x)$ is the shadow cost encoding the scarcity value for the oil producer when his reserves are at level $x$. The equilibrium production rates in the dynamic game are given by

$$
\bar{q}_{i}^{\star}(x(t))=q_{i}^{\star}\left(v_{0}^{\prime}(x(t)), s\right), \quad i=0, \ldots, N-1 .
$$

As these are determined by the value function $v_{0}$, we will not study the other players' value functions $w_{i}$. 


\subsection{Blockading Points}

When oil is plentiful, that is, $x$ is large, we expect the cost of energy production from alternative technologies to be too cost prohibitive to compete with the oil producer, and so he is the only active player. This is because the oil producer's scarcity cost $v_{0}^{\prime}(x) \rightarrow 0$ as $x \rightarrow \infty$, and so, when reserves are large, it is small compared to the cost of the alternative technologies. At the other extreme, when $x$ is small, we expect all $N$ producers to be active because the scarcity cost rises as reserves fall. Therefore, as reserves decline, we will go from monopoly, through duopoly to the full oligopoly with $N$ players. The reserve level at which Player $n$ enters is denoted $x_{b}^{n}$. Similarly, the time at which Player $n$ enters is denoted by $t_{b}^{n}$. We illustrate this intuition in Figure 5.

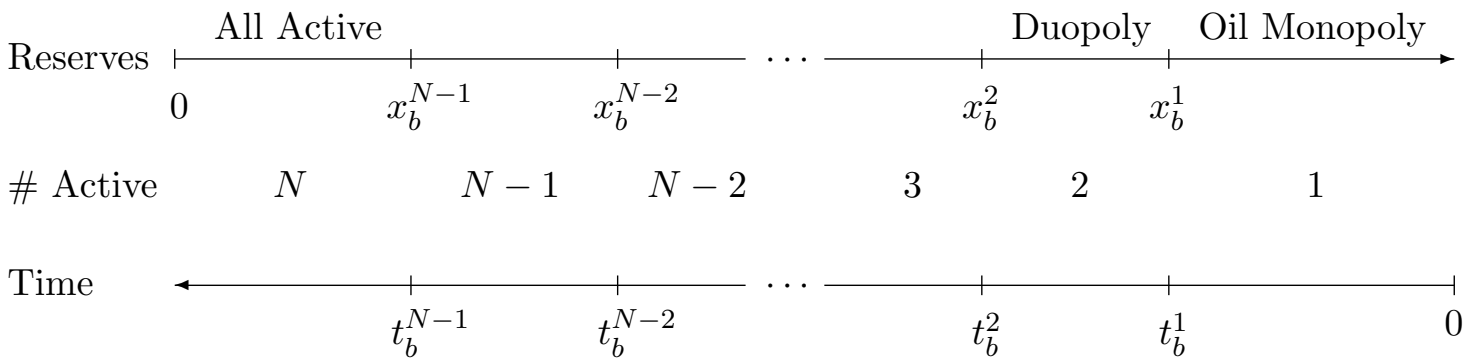

Figure 5: Blockading intuition

In other words, we expect for $x \in\left(0, x_{b}^{N-1}\right)$ that there are $N$ active firms in the sense that they all produce a positive quantity. For $x \in\left(x_{b}^{n}, x_{b}^{n-1}\right)$, there are $n$ active firms and $N-n$ inactive firms in the sense that in equilibrium they produce a zero quantity over this range of reserves. As reserves decline, $x_{b}^{n}$ denotes the point at which Firm $n$ begins production and enters the market. Or, coming from the left, $x_{b}^{n}$ is the level of reserves above which Firm $n$ is blockaded from the market. We shall see that the blockading points $x_{b}^{n}$ depend on the costs of the alternative technologies, and in fact, if these costs are small enough, it is possible to have $x_{b}^{n}=\infty$ for some values of $n$. We first introduce the notation

$$
\rho_{n}=\frac{1+S^{(n-1)}}{n} .
$$

Assumption 5.1. The vector of costs $s$ is such that $s_{N-1}<\rho_{N-1}$.

This is the condition from Lemma 4.1 that implies that Firm $N-1$ is active in the equilibrium at $x=0$. Furthermore, it is clear that, as Firm $N-1$ has the highest cost, all $N-1$ alternative technology firms will be active in the equilibrium at $x=0$. In fact as we assume for the $N-1$-player game that is played repeatedly after oil runs out is such that $n^{\star}=N-1$, then Proposition A.1 tells us that the sequence $\left\{Q_{H}^{\star, n}\right\}$ is strictly increasing and so, from Lemma 4.1, we have

$$
s_{n}<\rho_{n}, \quad \text { for all } n=1, \ldots, N-1 .
$$




\subsection{Low Oil Reserves: All Firms Active}

For $x(t)$ small enough, as we have stated above, all firms will be active in the sense that $\bar{q}_{i}^{\star}(x(t))>0$ for all $i=0, \ldots, N-1$. The blockading point $x_{b}^{N-1}$ is defined by

$$
x_{b}^{N-1}=\inf \left\{x>0: \bar{q}_{N-1}^{\star}(x)=0\right\} .
$$

We define $v^{(N)}(x)=v_{0}(x)$ for $x \in\left(0, x_{b}^{N-1}\right)$ where there are a total of $N$ active firms. Then we have the following explicit formulas for $v^{(N)}$ and the equilibrium production rates.

Proposition 5.1. For $x \in\left(0, x_{b}^{N-1}\right)$, Player 0 's value function is given by

$$
v^{(N)}(x)=\frac{1}{r}\left(\frac{1+S^{(N-1)}}{N+1}\right)^{2}(1+\mathbf{W}(\theta(x)))^{2},
$$

with

$$
\theta(x)=-e^{-\mu_{N} x-1} \quad \text { and } \quad \mu_{N}=\frac{2 r}{\rho_{N}}\left(\frac{1}{2}+\frac{1}{2 N}\right)^{2},
$$

and where $\mathbf{W}(\cdot)$ is the Lambert $\mathbf{W}$ function defined as the inverse function of $x e^{x}$, restricted to the range $[-1, \infty)$ and the domain $\left[-e^{-1}, \infty\right)$. Moreover,

$$
\begin{aligned}
& \bar{q}_{0}^{\star}(x(t))=\frac{1}{(N+1)}\left(1-N v^{(N)^{\prime}}(x(t))+S^{(N-1)}\right), \\
& \bar{q}_{i}^{\star}(x(t))=\frac{1}{(N+1)}\left(1-(N+1) s_{i}+v^{(N)^{\prime}}(x(t))+S^{(N-1)}\right), \quad i=1, \ldots, N-1,
\end{aligned}
$$

where $v^{(N)^{\prime}}(x)=-\rho_{N} \mathbf{W}(\theta(x))$.

The following lemma will be used in the proof.

Lemma 5.1. The $O D E\left(\alpha-v^{\prime}\right)^{2}=\kappa v$ on $\{x>0\}$, with $v(0)=v_{0} \geq 0, \alpha, \kappa>0$, has the solution

$$
v(x)=\frac{\alpha^{2}}{\kappa}(1+\mathbf{W}(\theta(x)))^{2},
$$

where

$$
\theta(x)=\beta e^{\beta} e^{-\kappa x / 2 \alpha} \quad \text { and } \quad \beta=-1+\frac{\sqrt{\kappa v_{0}}}{\alpha}
$$

Proof. This follows by direct substitution of Expression (33) into the ODE and the fact that $\mathbf{W}^{\prime}(z)=\mathbf{W}(z) /(z(1+\mathbf{W}(z)))$.

Proof of Prop. 5.1. From Proposition A.1, the Nash equilibrium for the static game with $N$ active players with costs $\left(s_{0}, s\right)$ is given by

$$
q_{i}^{\star}\left(s_{0}, s\right)=\frac{1}{N+1}\left(1-N s_{i}+\sum_{j=0, j \neq i}^{N-1} s_{j}\right), \quad i=0, \ldots, N-1 .
$$


The ODE (27) in the interval $x \in\left(0, x_{b}^{N-1}\right)$ becomes

$$
r v^{(N)}=\frac{1}{(N+1)^{2}}\left(1+S^{(N-1)}-N v^{(N)^{\prime}}\right)^{2}
$$

for which we can use Lemma 5.1 to give Expression (30). The equilibrium policies in Equation (32) follow from Expression (35) with $s_{0}=v^{(N)^{\prime}}(x)$.

Next we find the blockading point $x_{b}^{N-1}$, the point at which Firm $N-1$ would no longer wish to produce a positive quantity, and therefore would no longer be active. To simplify the remainder of the results, we first introduce the notation, for $n=1, \ldots, N-1$,

$$
\delta_{n}=(n+1) s_{n}-\left(1+S^{(n-1)}\right) .
$$

Proposition 5.2. The last blockading point is given by:

$$
x_{b}^{N-1}=\frac{1}{\mu_{N}}\left[-1+\frac{\delta_{N-1}}{\rho_{N}}-\log \left(\frac{\delta_{N-1}}{\rho_{N}}\right)\right],
$$

provided $\delta_{N-1}>0$, otherwise $x_{b}^{N-1}=\infty$.

The following lemma is useful for the calculation.

Lemma 5.2. For a given constant $H<0$, the solution to $\mathbf{W}(\theta(x))=H$, where $\theta(x)$ is as in Equation (34), is given by

$$
x=\frac{2 \alpha}{\kappa}\left(\beta-H-\log \left(\frac{H}{\beta}\right)\right) .
$$

Proof. By definition, $\mathbf{W}(Y)=H$ if and only if $H e^{H}=Y$. Moreover, $\theta(x)=z$ if and only if $x=\frac{2 \alpha}{\kappa}(\beta-\log (z / \beta))$. Hence, $\mathbf{W}(\theta(x))=H$ if and only if $\theta(x)=H e^{H}$, which results in Equation (38).

Proof of Prop. 5.2. From the definition (29), we solve for $x$ such that $\bar{q}_{N-1}^{\star}(x)=0$. From Expression (32), the necessary equation to solve is

$$
\mathbf{W}\left(\theta\left(x_{b}^{N-1}\right)\right)=-\frac{\delta_{N-1}}{\rho_{N}}
$$

and Equation (37) follows from Lemma 5.2.

\subsection{Higher Reserve Levels: some firms blockaded}

We denote by $v^{(n)}\left(x-x_{b}^{n}\right)$ the value function of the oil producer when there are $n$ total active firms. To this end, we define $v^{(n)}\left(x-x_{b}^{n}\right)=v_{0}(x)$ for $x \in\left[x_{b}^{n}, x_{b}^{n-1}\right]$, where we have included in the domain the end points of transition in the number of players. From our characterization of $x_{b}^{N-1}$ in Equation (39), we have

$$
v^{(N-1)}(0)=v^{(N)}\left(x_{b}^{N-1}\right)=\frac{1}{r}\left(s_{N-1}-\delta_{N-1}\right)^{2} .
$$


Proposition 5.3. Suppose that for $n \in\{2, \ldots, N-1\}, x_{b}^{n}<\infty$. If $\delta_{n-1}>0$, then

$$
x_{b}^{n-1}=x_{b}^{n}+\frac{1}{\mu_{n}}\left[\log \left(\frac{\delta_{n}}{\delta_{n-1}}\right)-\frac{(n+1)\left(s_{n}-s_{n-1}\right)}{\rho_{n}}\right] \text {, }
$$

otherwise $x_{b}^{n-1}=\infty$. For $x \in\left[x_{b}^{n}, x_{b}^{n-1}\right)$,

$$
v^{(n)}\left(x-x_{b}^{n}\right)=\frac{1}{r}\left(\frac{1+S^{(n-1)}}{n+1}\right)^{2}\left(1+\mathbf{W}\left(\theta\left(x-x_{b}^{n}\right)\right)\right)^{2},
$$

where

$$
\theta\left(x-x_{b}^{n}\right)=-\frac{\delta_{n}}{\rho_{n}} e^{-\mu_{n}\left(x-x_{b}^{n}\right)-\delta_{n} / \rho_{n}} \quad \text { and } \quad \mu_{n}=\frac{2 r}{\rho_{n}}\left(\frac{1}{2}+\frac{1}{2 n}\right)^{2}
$$

Proof. First, suppose

$$
v^{(n+1)}\left(x_{b}^{n}-x_{b}^{n+1}\right)=\frac{1}{r}\left(s_{n}-\delta_{n}\right)^{2},
$$

(where for $n=N-1$, we identify $x_{b}^{N}=0$ ), and suppose $\delta_{n}>0$. Then, for $x \in\left(x_{b}^{n}, x_{b}^{n-1}\right.$ ), the ODE (27) becomes

$$
r v^{(n)}\left(x-x_{b}^{n}\right)=\frac{1}{(n+1)^{2}}\left(1+S^{(n-1)}-n v^{(n)^{\prime}}\left(x-x_{b}^{n}\right)\right)^{2} .
$$

Solving Equation (44) with the boundary condition $v^{(n)}(0)=v^{(n+1)}\left(x_{b}^{n}-x_{b}^{n+1}\right)$, given in Equation (43), and using Lemma 5.1, gives Equation (42).

The equation that specifies the blockading point $x_{b}^{n-1}$ is

$$
\bar{q}_{n-1}^{\star}\left(x_{b}^{n-1}\right)=\frac{1}{n+1}\left(1-(n+1) s_{n-1}+v^{(n)^{\prime}}\left(x_{b}^{n-1}-x_{b}^{n}\right)+S^{(n-1)}\right)=0 .
$$

Using Equation (42) in the ODE (44) gives

$$
v^{(n)^{\prime}}\left(x-x_{b}^{n}\right)=-\rho_{n} \mathbf{W}\left(\theta\left(x-x_{b}^{n}\right)\right),
$$

which leads to solving

$$
\mathbf{W}\left(\theta\left(x_{b}^{n-1}-x_{b}^{n}\right)\right)=-\frac{\delta_{n-1}}{\rho_{n}} .
$$

From Lemma 5.2, there is a unique, real solution given by Equation (41) if and only if $\delta_{n-1}>0$. If this latter condition does not hold, then $x_{b}^{n-1}=\infty$.

Substituting from Equation (45) into Equation (42), we compute

$$
v^{(n)}\left(x_{b}^{n-1}-x_{b}^{n}\right)=\frac{1}{r}\left(s_{n-1}-\delta_{n-1}\right)^{2},
$$

which is identical to Equation (43) with $n$ replaced by $n-1$.

Finally, with $n=N-1$, we see from Equation (40) that Equation (43) is satisfied, and from Proposition 5.2, $x_{b}^{N-1}<\infty$ if and only if $\delta_{N-1}>0$. Therefore, from Equation (46), Equation (43) holds for $n=N-2$; and from the above, $\delta_{n}>0$ holds for $n=N-2$, and we complete by induction. 
The proposition shows that $x_{b}^{n-1}<\infty$ if and only if $x_{b}^{n}<\infty$ and $s_{n-1}>\left(1+S^{(n-2)}\right) / n$. That is, Player $n-1$ will be blockaded for large enough $x$, if his cost is high relative to his lower cost competitors'.

Remark 5.1. Note that, combining the results of the prior proposition with Equation (28), the interesting range of costs where there is a full $N-1$ active player oligopoly at $x=0$ and a monopoly for the oil producer for $x$ large enough is

$$
\frac{1+S^{(n-1)}}{n+1}<s_{n}<\frac{1+S^{(n-1)}}{n}, \quad \text { for all } n=1, \ldots, N-1,
$$

or using the definition in Equation (36),

$$
0<\delta_{n}<s_{n}, \quad \text { for all } n=1, \ldots, N-1 .
$$

Remark 5.2. The associated Hamilton-Jacobi equation for $w_{i}$ is given by

$$
r w_{i}=-\bar{q}_{0}^{\star} w_{i}^{\prime}+G_{i}\left(v_{0}^{\prime}, s\right), \quad w_{i}(0)=G_{i}(1, s) / r .
$$

It is possible to solve for these functions explicitly. However, the strategies of the firms depend only on $x$ through $v_{0}$, and therefore these functions are not necessary for the analysis of the game. We illustrate the methodology for $x \in\left(0, x_{b}^{N-1}\right)$.

For $i=1, \ldots, N-1$ and $x \in\left(0, x_{b}^{N-1}\right)$, the ODE to solve is

$$
r w_{i}=-\frac{1}{(N+1)}\left(1-N v^{(N)^{\prime}}+S^{(N-1)}\right) w_{i}^{\prime}+\frac{1}{(N+1)^{2}}\left(1-(N+1) s_{i}+v^{(N)^{\prime}}+S^{(N-1)}\right)^{2} .
$$

We make the change of variable $\xi=v^{(N)^{\prime}}(x)$, and set $w_{i}(x)=f(\xi)$. Using Equation (49) and properties of $v^{(N)}$, we find

$$
f_{i}^{\prime}-\left(\frac{2 N}{N+1}\right) \frac{1}{\xi} f_{i}=-\frac{2 N}{(N+1)^{3} r \xi}\left(\xi-(N+1) s_{i}+1+S^{(N-1)}\right)^{2}
$$

with boundary condition

$$
f_{i}\left(\rho_{N}\right)=\frac{1}{r} G_{i}(1, s)=\frac{1}{r N^{2}}\left(1-N s_{i}+S^{(N-1)}\right)^{2} .
$$

Solving this ODE gives

$$
f_{i}(\xi)=K_{i}\left(\frac{\xi}{\rho_{N}}\right)^{\frac{2 N}{N+1}}-\frac{2 N}{(N+1)^{3} r}\left[\left(\frac{N+1}{2}\right) \xi^{2}+\frac{2(N+1) h_{i}}{2 N-1} \xi-\frac{(N+1) h_{i}^{2}}{2 N}\right],
$$

where

$$
\begin{aligned}
h_{i} & =(N+1) s_{i}-\left(1+S^{(N-1)}\right) \\
K_{i} & =\frac{\left(1+S^{(N-1)}\right)\left[\left(1+S^{(N-1)}\right)(2 N+1)(N-1)+2 N(N+1) s_{i}\right]}{N^{2}(2 N-1)(N+1)^{2} r} .
\end{aligned}
$$

This holds for $\xi \in\left(\rho_{N}, \delta_{N-1}\right)$ or, equivalently, $x \in\left(0, x_{b}^{N-1}\right)$. One would then continue to the next blockading region and solve a nearly identical ODE with a different initial condition. This can be done explicitly, but as we shall not use these results, we omit the details here. 


\subsection{Properties of the Value Function}

We have assumed throughout that the value function for the oil producer is continuous at the blockading points. This assumption has driven the boundary conditions in the ODEs that we have used to find the corresponding $v^{(n)}$. We show in this section that the value function also has a continuous first derivative at the boundary points, but that the second derivative is discontinuous at these points. As the first derivative of $v_{0}$ determines the market price, this implies that the market price is continuous at the blockading points, but the rate of change over time of the market price has discontinuities.

Proposition 5.4. For a given $n \geq 2$, we have

$$
v^{(n)^{\prime}}\left(x_{b}^{n-1}-x_{b}^{n}\right)=v^{(n-1)^{\prime}}(0) .
$$

In other words, the first derivative of $v_{0}$ is continuous at $x_{b}^{n-1}$.

Proof. By construction, we have

$$
v^{(n)}\left(x_{b}^{n-1}-x_{b}^{n}\right)=v^{(n-1)}(0)=\frac{1}{r}\left(s_{n-1}-\delta_{n-1}\right)^{2} .
$$

The relevant ODEs for these two functions are

$$
r v^{(m)}=\frac{1}{(m+1)^{2}}\left(1+S^{(m-1)}-m v^{(m)^{\prime}}\right)^{2}
$$

for $m, n-1$. This implies

$$
\begin{aligned}
v^{(n)^{\prime}\left(x_{b}^{n-1}-x_{b}^{n}\right)} & =\frac{1+S^{(n-1)}-(n+1) \sqrt{r v^{(n)}\left(x_{b}^{n-1}-x_{b}^{n}\right)}}{n} \\
& =\frac{1}{n}\left[1+S^{(n-1)}-(n+1)\left(1+S^{(n-2)}-(n-1) s_{n-1}\right)\right] \\
& =\delta_{n-1}, \quad \text { and } \\
v^{(n-1)^{\prime}}(0) & =\frac{1+S^{(n-2)}-n \sqrt{r v^{(n-1)}(0)}}{n-1} \\
& =\frac{1}{n-1}\left[1+S^{(n-2)}-n\left(1+S^{(n-2)}-(n-1) s_{n-1}\right)\right] \\
& =\delta_{n-1} .
\end{aligned}
$$

Proposition 5.5. For a given $n \geq 2$, we have

$$
v^{(n)^{\prime \prime}}\left(x_{b}^{n-1}-x_{b}^{n}\right)>v^{(n-1)^{\prime \prime}}(0)
$$

In other words, there is a downward jump when moving in the direction of larger $x$ in the second derivative of $v_{0}$ at the point $x_{b}^{n-1}$. 
Proof. Differentiating the ODE given in Equation (50) gives

$$
r v^{(m)^{\prime}}=\frac{-2 m}{(m+1)^{2}}\left(1+S^{(m-1)}-m v^{(m)^{\prime}}\right) v^{(m)^{\prime \prime}}
$$

and rearranging terms, we find

$$
v^{(m)^{\prime \prime}}=\frac{-(m+1)^{2} r v^{(m)^{\prime}}}{2 m\left(1+S^{(m-1)}-m v^{(m)^{\prime}}\right)},
$$

for $m, n-1$. The proof of Proposition 5.4 shows that $v^{(n)^{\prime}}\left(x_{b}^{n-1}-x_{b}^{n}\right)=v^{(n-1)^{\prime}}(0)=\delta_{n-1}$, therefore

$$
v^{(n)^{\prime \prime}}\left(x_{b}^{n-1}-x_{b}^{n}\right)-v^{(n-1)^{\prime \prime}}(0)=\frac{r}{2 n^{2}\left(n^{2}-1\right)}\left(\frac{\delta_{n-1}}{s_{n-1}-\delta_{n-1}}\right)>0,
$$

where the positivity of the term in parentheses comes from Equation (48).

Along similar lines, we also find that a modified version of Hotelling's rule holds.

Proposition 5.6. For $n \in\{1, \ldots, N\}$, for $x \in\left(x_{b}^{n}, x_{b}^{n-1}\right)$, (we identify $x_{b}^{N}=0$ and $x_{b}^{0}=\infty$ ),

$$
\frac{d}{d t} v^{(n)^{\prime}}\left(x(t)-x_{b}^{n}\right)=\left(\frac{1}{2}+\frac{1}{2 n}\right) r v^{(n)^{\prime}}\left(x(t)-x_{b}^{n}\right) .
$$

Proof. Let $x \in\left(x_{b}^{n}, x_{b}^{n-1}\right)$. We differentiate the ODE in Equation (50) which gives

$$
r v^{(n)^{\prime}}=\frac{2 n}{n+1}\left[\frac{1}{n+1}\left(1-n v^{(n)^{\prime}}+S^{(n-1)}\right)\left(-v^{(n)^{\prime \prime}}\right)\right] .
$$

Furthermore, we recall from Equations (25) and (31)

$$
\frac{d x}{d t}=-\bar{q}_{0}^{\star}(x(t))=\frac{-1}{n+1}\left(1-n v^{(n)^{\prime}}+S^{(n-1)}\right) .
$$

Then,

$$
\begin{aligned}
\frac{d}{d t} v^{(n)^{\prime}}\left(x(t)-x_{b}^{n}\right) & =v^{(n)^{\prime \prime}}\left(x(t)-x_{b}^{n}\right)\left(-\bar{q}_{0}^{\star}(x(t))\right) \\
& =\frac{1}{n+1}\left(1-n v^{(n)^{\prime}}+S^{(n-1)}\right)\left(-v^{(n)^{\prime \prime}}\right) \\
& =\left(\frac{1}{2}+\frac{1}{2 n}\right) r v^{(n)^{\prime}}
\end{aligned}
$$

The result, Equation (51), coincides with the classical Hotelling rule (see [15]) for $n=1$, which states that, for a monopolist, the marginal value grows (exponentially) at the discount rate $r$. 
This result on the growth rate of the marginal value has implications for the price function over time due to the dependence of price on the marginal value. First, we define $P^{(n)}\left(x-x_{b}^{n}\right)$ to be the market price for $x \in\left(x_{b}^{n}, x_{b}^{n-1}\right)$. The general form of the price given in Equation (26) implies

$$
\begin{aligned}
P^{(n)}\left(x(t)-x_{b}^{n}\right) & =1-\bar{q}_{0}^{\star}(x(t))-\sum_{i=1}^{n-1} \bar{q}_{i}^{\star}(x(t)) \\
& =1-\frac{1}{n+1}\left(1-n v^{(n)^{\prime}}+S^{(n-1)}\right)-\frac{1}{n+1} \sum_{i=1}^{n-1}\left(1-(n+1) s_{i}+v^{(n)^{\prime}}+S^{(n-1)}\right) \\
& =\frac{1}{n+1}\left(1+v^{(n)^{\prime}}+S^{(n-1)}\right) .
\end{aligned}
$$

Note, we can use the proof of Proposition 5.4 to see that $P^{(n)}\left(x_{b}^{n-1}-x_{b}^{n}\right)=s_{n-1}$. That is, the blockading point $x_{b}^{n-1}$ is exactly the point at which the market price equals the marginal cost of Firm $n-1$.

We define the function $P(t)=P^{(n)}\left(x(t)-x_{b}^{n}\right)$ for $t$ such that $x(t) \in\left(x_{b}^{n}, x_{b}^{n-1}\right)$. This function differs from Equation (26) as it is a function of the time variable, $t$, rather than of oil reserves, $x(t)$. We further define the blockading times

$$
\begin{aligned}
& t_{b}^{1}=\inf \left\{t>0: x(t)=x_{b}^{1}\right\}=\inf \left\{t>0: P(t)=s_{1}\right\}, \\
& t_{b}^{n}=\inf \left\{t>t_{b}^{n-1}: P(t)=s_{n}\right\}, \quad n=2, \ldots, N-1 .
\end{aligned}
$$

Proposition 5.7. For $n \in\{2, \ldots, N\}$, for $t$ such that $x(t) \in\left(x_{b}^{n}, x_{b}^{n-1}\right)$, (we identify $x_{b}^{N}=0$ ),

$$
P(t)=\frac{1+S^{(n-1)}}{n+1}+\left(\frac{\delta_{n-1}}{n+1}\right) \exp \left\{\left(\frac{1}{2}+\frac{1}{2 n}\right) r\left(t-t_{b}^{n-1}\right)\right\}
$$

and for $n=1$, i.e. for $t$ such that $x(t) \in\left(x_{b}^{1}, x(0)\right)$,

$$
P(t)=\frac{1}{2}+\left(P(0)-\frac{1}{2}\right) e^{r t}
$$

where the price at $t=0$ is given by

$$
P(0)=\frac{1}{2}\left(1-\mathbf{W}\left(\left(1-2 s_{1}\right) e^{-2 r\left(x(0)-x_{b}^{1}\right)+\left(1-2 s_{1}\right)}\right)\right) .
$$

Proof. We differentiate Equation (52) and apply Proposition 5.6 to obtain

$$
\frac{d}{d t} P^{(n)}\left(x(t)-x_{b}^{n}\right)=\frac{1}{n+1} \frac{d}{d t} v^{(n)^{\prime}}\left(x(t)-x_{b}^{n}\right)=\left(\frac{1}{2 n}\right) r v^{(n)^{\prime}}\left(x(t)-x_{b}^{n}\right) .
$$

We then arrange Equation (52) to express $v^{(n)^{\prime}}$ in terms of $P^{(n)}$, insert into Equation (55), and use the definition of $P(t)$ to obtain

$$
\frac{d}{d t} P(t)=\left(\frac{1}{2}+\frac{1}{2 n}\right) r\left(P(t)-\frac{1+S^{(n-1)}}{n+1}\right) .
$$

Solving this ODE, over the relevant ranges, with the initial conditions $P\left(t_{b}^{n-1}\right)=s_{n-1}$, gives Equations (53) and (54). 
Proposition 5.7 shows that in between blockading points, the price starts at the cost of the previous firm to enter, and increases exponentially to the cost of the firm with the next highest cost. The rate of growth depends on the number of active firms, and indirectly on the costs of all the firms in the game. Furthermore, the expression in (53) provides an explicit representation of the blockading times.

Proposition 5.8. For $n \in\{2, \ldots, N-1\}$, the time at which Firm $n$ enters the game is given by

$$
t_{b}^{n}=t_{b}^{n-1}+\frac{2 n}{(n+1) r} \log \left(\frac{\delta_{n}}{\delta_{n-1}}\right)
$$

and for $n=1$ by

$$
t_{b}^{1}=\frac{1}{r} \log \left(\frac{s_{1}-\frac{1}{2}}{P(0)-\frac{1}{2}}\right) .
$$

Comparing Equation (57) with Equation (41), we notice that the exact same condition required for the blockading times to be well-defined is that which makes the blockading points well-defined.

As time increases, the game moves from larger values of $x$ to lesser values of $x$ as oil reserves are depleted. Therefore, moving in the positive direction of time, the price transitions from $P^{(n-1)}$ to $P^{(n)}$ at the point $x_{b}^{n-1}$, and the price function is continuous at this point; however, the same cannot be said about the time derivative of the price.

Proposition 5.9. For $n \in\{2, \ldots, N-1\}$, there is a jump down in $(d / d t) P(t)$ at each blockading time. In other words,

$$
\lim _{t \downarrow t_{b}^{n-1}} \frac{d}{d t} P(t)=\frac{d}{d t} P^{(n)}\left(x_{b}^{n-1}-x_{b}^{n}\right)<\frac{d}{d t} P^{(n-1)}(0)=\lim _{t \uparrow t_{b}^{n-1}} \frac{d}{d t} P(t) .
$$

Proof. From Equation (56) or Equation (55), and the continuity of the market price,

$$
\begin{aligned}
\frac{d}{d t} P^{(n)}\left(x_{b}^{n-1}-x_{b}^{n}\right) & -\frac{d}{d t} P^{(n-1)}(0) \\
& =\frac{r}{2}\left[\left(1+\frac{1}{n}\right)\left(s_{n-1}-\frac{1+S^{(n-1)}}{n+1}\right)-\left(1+\frac{1}{n-1}\right)\left(s_{n-1}-\frac{1+S^{(n-2)}}{n}\right)\right] \\
& =-\frac{r \delta_{n-1}}{2 n(n-1)}<0
\end{aligned}
$$

where the negativity comes from the fact that $\delta_{n-1}>0$ (see Equation (48)).

This proposition says that the time derivative of price jumps down at each blockading point. The size of the jump at $x_{b}^{n-1}$ depends on $n$, as well as the gap in Equation (47) for $s_{n-1}$.

\subsection{Numerical Example}

In the following illustrations, we set $r=1$. Figure 6 displays the resulting trajectory of $x(t)$, production rates, and market price for $N=10$, and $s_{1}=0.51, s_{2}=0.52, \ldots, s_{9}=0.59$, which 
satisfy the condition (47). The vertical lines are the times associated with the blockading points, i.e. from the left to right, at each vertical line the number of active players increases by one. At the far right, once the oil reserves hit zero, we see that production rates and market price are constant, because in this region the alternative technology producers are the only active firms and they play an infinitely repeated static game. We observe the behavior in the remark prior to Proposition 5.7 in the market price figure. Namely, each vertical line, which represents the entry of a firm, is exactly where the market price crosses the horizontal line at that firm's cost. In other words, the first blockading point, where the game transitions from a monopoly to duopoly, occurs exactly where $P=c_{1}=0.51$.
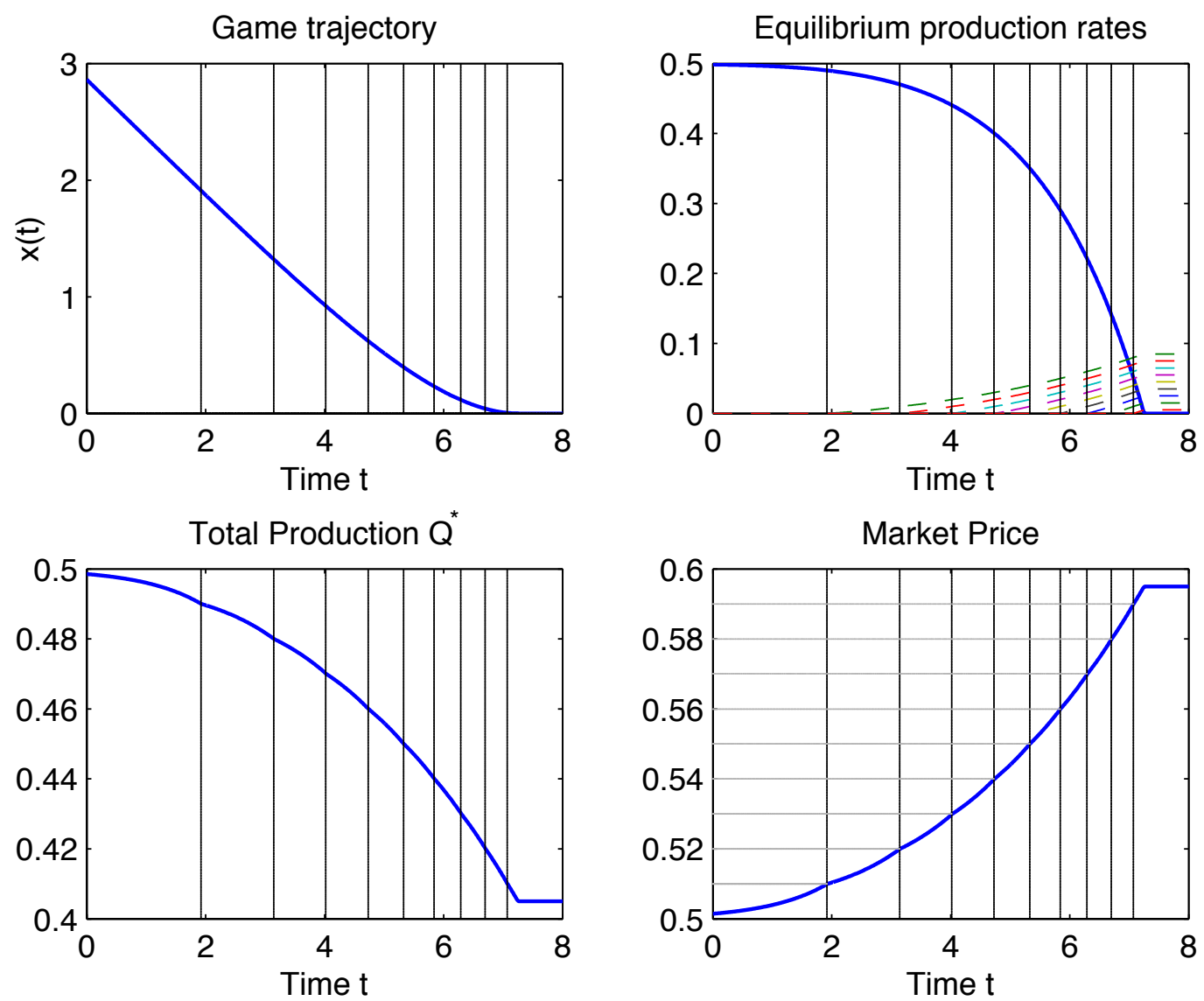

Figure 6: Numerical example with 10 firms. In the upper-right figure, the increasing dashed lines represent the production rates of the renewable producers.

The time derivative of market price is displayed in Figure 7. We see that the derivative is everywhere positive, and at the blockading points, the derivative jumps down and then increases, exactly as described above. Therefore, price is strictly increasing over time, but the rate of the increase is lower immediately after a new firm enters the market. The derivative jumps to zero at the point where oil reserves are fully depleted. This is because the price is a constant in the equilibrium that involves only the alternative technology firms.

In energy production, costs of using various fuels or technologies may occur in groups, with hydro 


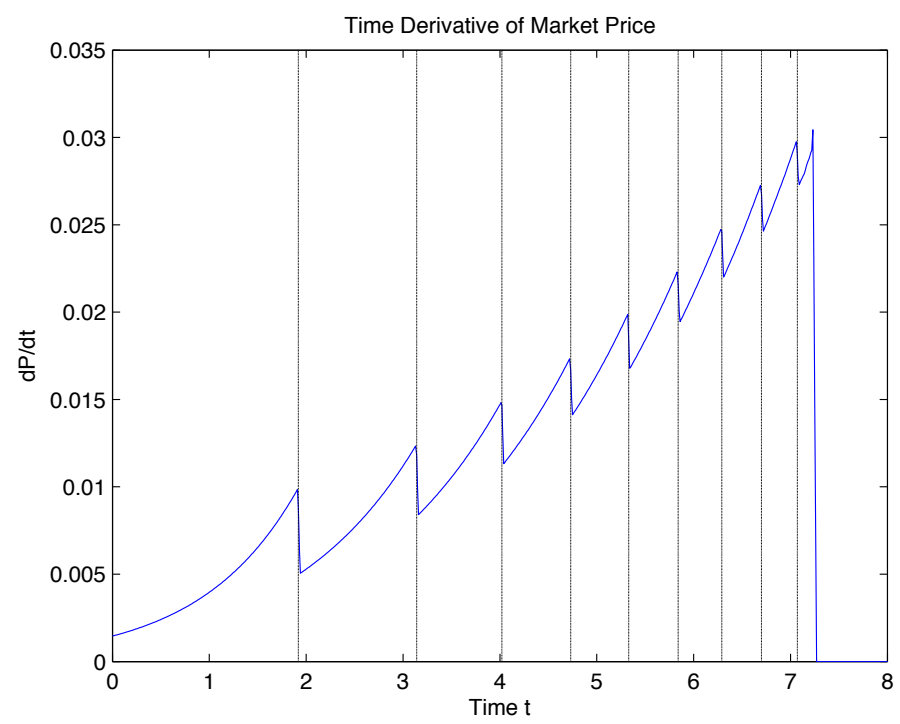

Figure 7: Time derivative of market price

and geothermal quite low, biomass and on-shore wind somewhat higher, and solar and tidal rather higher still. In Figure 8, we plot the trajectory of oil reserves, production rates, and market price, except here we set $s=(0.51,0.52,0.57,0.58,0.62,0.63)$, which corresponds to $N=7$. These costs are such that we have 3 different groups of technologies; within each group, there are two firms with a cost difference equal to 0.01 . We can see from the game trajectory, for instance, that the same absolute cost difference can lead to different sized intervals of time between entries. In particular, the amount of time between the entry of Firm 2 after Firm 1 is much greater than the amount of time between the entry of Firm 6 after Firm 5 (recall that the oil producer is Firm 0). Thus the transition to alternative sources occurs more rapidly over time.

\section{Conclusion}

We have analyzed the number of firms who are active in equilibrium in Cournot type and Bertrand type oligopolies in the case of both homogeneous and differentiated goods. Moreover, we have considered crucially the case where firms have asymmetric costs. Our main finding is that for a given type of good structure (i.e. either homogeneous or differentiated) Cournot markets have more active firms than the corresponding Bertrand market. Further, the differentiated market has more active firms than the corresponding homogeneous market for both Cournot and Bertrand type oligopolies. We find that the degree of product differentiation is thus crucial for the determination of the natural market size.

The essential result is that Bertrand markets give consumers less choices of goods, but they pay lower prices for those goods. On the other hand, Cournot markets give consumers an increased amount of variety, but at the cost of paying higher prices for those goods. This analysis has implications in the application of antitrust laws, in particular the use of the Herfindahl-Hirschman Index (HHI) by the Department of Justice in assessing the legality of a proposed merger. The above results translate to the statement that, ceteris paribus, Cournot markets have a lower HHI than 

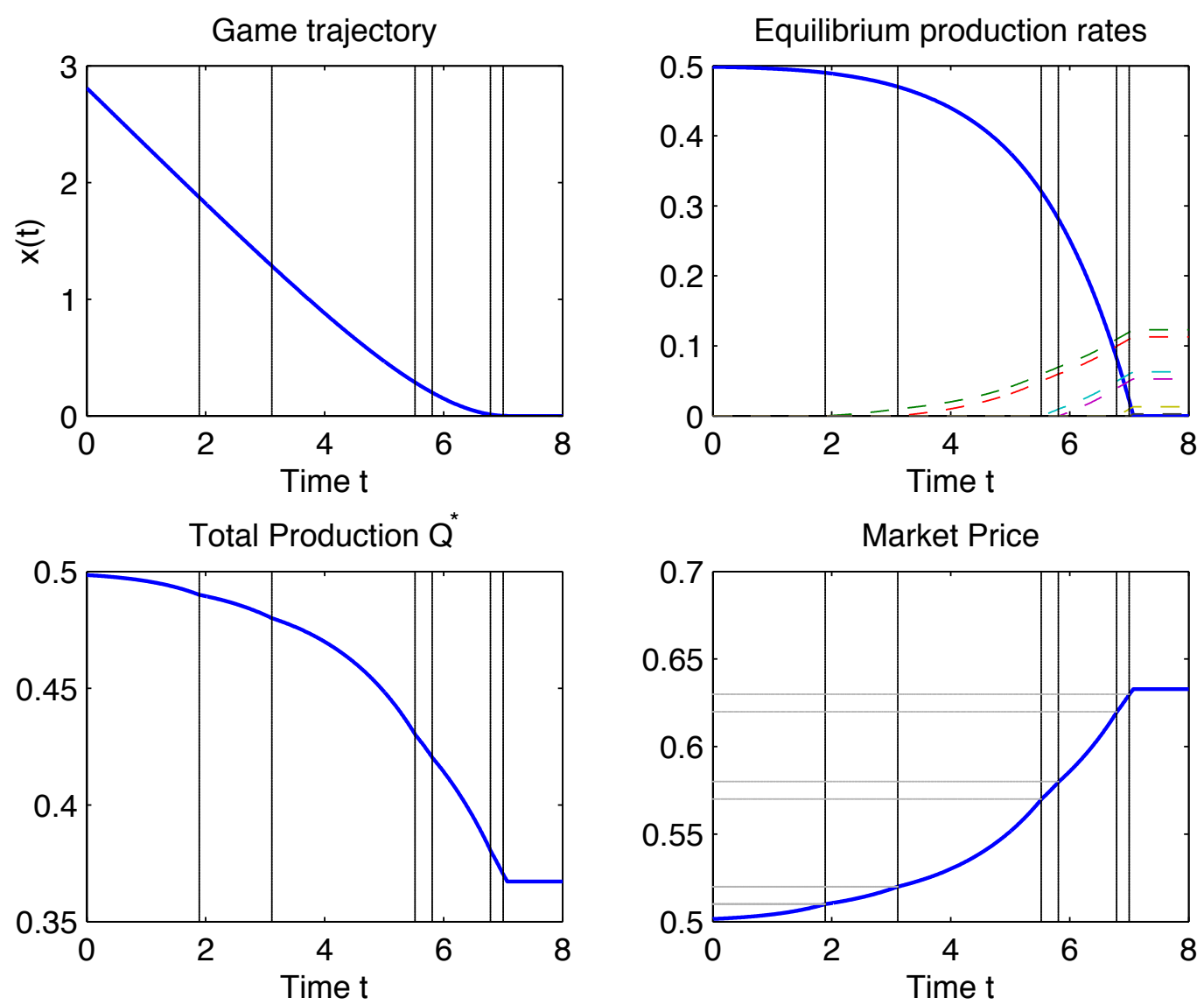

Figure 8: Numerical example with 7 firms.

Bertrand markets. However, in terms of competition, which this index is supposed to assess, one can strongly argue that Cournot markets are less competitive than Bertrand markets. Although this index is not meant to be comparable across different types of markets, the use of such an index relies on the assumption that market symmetry and more active firms are always better. However, the strength of competition in the Bertrand model results in a monopoly providing the lowest price because of the potential competition of higher cost firms. Therefore, this analysis is a clear indicator that the HHI is an incomplete metric.

In a dynamic game, the number of active participants may change over time. In an energy production Cournot model for exhaustible resources, we have seen how renewable energy producers come in as oil reserves deplete. Under linear demands, it is possible to fully characterize the entry levels and times and the downward jumps in the rate of price change. In addition, we get an explicit extension of Hotelling's rule for monopolistic exhaustible resource prices to the oligopoly situation, when the competition is from higher cost inexhaustible suppliers. It remains to understand the blockading issue when there are multiple exhaustible suppliers since that involves strongly coupled systems of nonlinear PDEs with nonsmooth coefficients.

A further issue is the effect of uncertainty both in reserve levels and demand fluctuations. The nonrandom ordinary differential game with linear demands is tractable as we saw in Section 5 and 
it is a goal to adapt its clean results to incorporate stochasticity.

\section{A Static Homogeneous Oligopolies}

\section{A.1 Cournot Competition}

Similar to the differentiated Cournot model, firms use quantity as their strategic variable to maximize Expression (7), where price $p_{i}^{H}(q)$ is given in Expression (5). We note that as price is only a function of the aggregate quantity $Q$, we in fact have the price function $P(Q)=p_{i}^{H}(Q)$, where the latter function is a slight abuse of notation of the previously defined price function. This model was studied extensively in the setting of general inverse demand functions in [13].

The calculation of the Nash equilibrium is identical to that of the differentiated case, except here instead of using the effective market price function $\bar{P}(Q)$, we use the actual market price function $P(Q)=p^{H}(Q)$. With this slight modification all of the results in Section 3.1 carry over to this setting, provided we set $\gamma=\beta$.

Setting $\gamma=\beta$ in Equation (13), the equilibrium total supply in the homogeneous model is given by

$$
Q_{H}^{\star, n}=\frac{n \alpha-S^{(n)}}{(n+1) \beta} .
$$

Here we use the subscript $H$ to stand for Homogeneous in order to discriminate this quantity from the one used in the differentiated Cournot game.

We then have the following result that fully characterizes the equilibrium. The proof follows the proof of Proposition 3.1 with $\gamma=\beta$.

Proposition A.1 (Corr 2.7 in [13]). The unique Nash equilibrium can be constructed as follows. Let $\bar{Q}_{H}^{\star}=\max \left\{Q_{H}^{\star, n} \mid 1 \leq n \leq N\right\}$. Then the unique Nash equilibrium quantities are given by

$$
q_{i}^{\star}(s)=\max \left\{\frac{\alpha-\beta \bar{Q}_{H}^{\star}-s_{i}}{\beta}, 0\right\}, \quad 1 \leq i \leq N .
$$

The number of active players in the unique equilibrium is $m=\min \left\{n \mid Q_{H}^{\star, n}=\bar{Q}_{H}^{\star}\right\}$.

\section{A.2 Bertrand Competition}

The homogeneous Bertrand case is perhaps the simplest and yet most difficult to analyze of the four models. As in the differentiated Bertrand case, the firms use price as their strategic variable in maximizing the profit function in (7) with quantity given by $q_{i}^{H}(p)$ in Expression (6). The difficulty arises from the non-smoothness of the demand function. If one firm sets a price slightly below the rest of the market, then they will receive all the demand. If another firm were to charge an even slightly lower price than this, then all of the demand would shift to this new lowest cost firm. This behavior makes the result seemingly very simple.

Consider first the case of a duopoly. We illustrate in Figure 9 the best response functions for the two firms, where $s_{1}<s_{2}$. The best response is to charge cost while your opponent is pricing below 
your cost, and then to price at your opponent's price minus a small amount, which we denote informally as $p^{\star}=\left(s_{2}-\epsilon, s_{2}\right)$. The reason for this is that if Firm 1 were to price strictly above $s_{2}$,

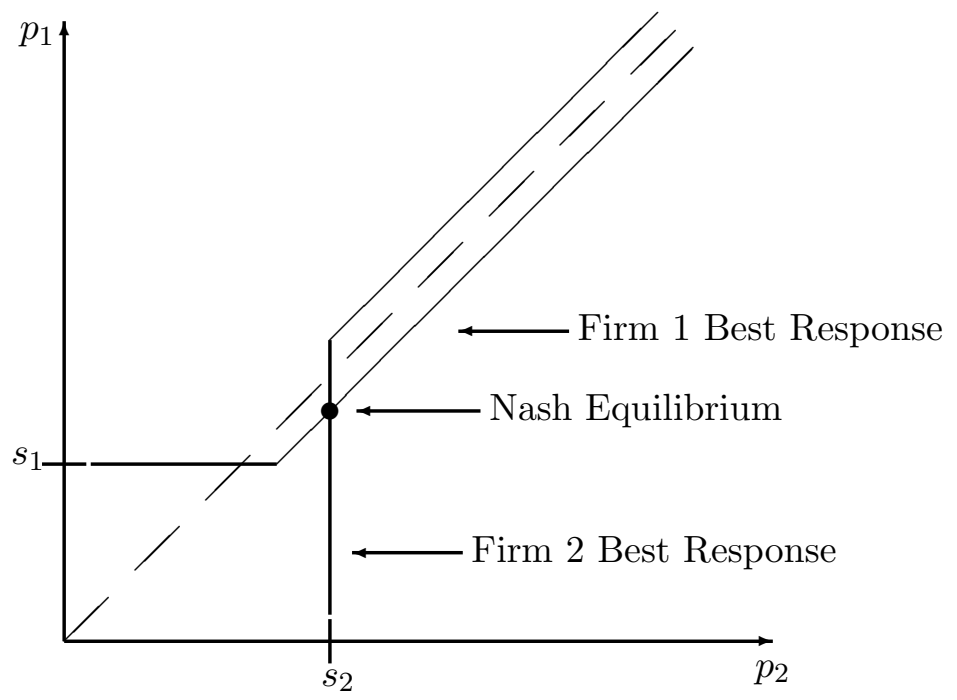

Figure 9: Best Response Functions for Homogeneous Bertrand Duopoly

by any amount, Firm 2 would have an incentive to deviate from sharing demand at this price by lowering price by a small amount and thereby capture the entire market at a price above cost. On the other hand, if Firm 2 were to price exactly at $s_{2}$, Firm 1 would have an incentive to deviate to a slightly lower price in order to avoid splitting demand in half. The same concept extends to $N$ firms in the sense that the lowest cost firm is the only one who ever receives demand from the market.

There is a subtle issue here as, for a fixed value of $\epsilon>0$, Firm 1 would always be better off decreasing $\epsilon$ by a small amount and therefore increasing profit. This is what has been referred to as the open-set problem, see [7] and references therein. We follow their solution of such a situation and assume that the equilibrium exists in the limiting sense of Firm 1 setting price equal to $s_{2}$ and obtaining the entire market demand at this price, while Firm 2 sells nothing and gets zero profit. Further, we have assumed throughout in a Bertrand market that any firm with zero demand in equilibrium at any price greater or equal to cost sets price equal to cost. Thus, we have an unique equilibrium (albeit in a limiting sense) of $p^{\star}=\left(s_{2}, s_{2}\right)$, with Firm 1 supplying the whole market.

If Firm 1 knew they were the only firm in the market, then they would charge their optimal monopoly price

$$
p_{M}^{\star}=\frac{1}{2}\left(\alpha+s_{1}\right) .
$$

This is greater than cost provided $s_{1}<\alpha$. If there are other firms in the market, Firm 1 will not always set this price in equilibrium, because if they did, their exist cases where other firms would have an incentive to undercut that price and capture all of the demand in the market. In fact, one can show that no equilibrium is sustainable with $p_{1}>s_{2}$, regardless of the number of firms in the market. Furthermore, for $\epsilon>0$ small enough, we have that the profit of Firm 1 from setting $p_{1}=s_{2}-\epsilon$ is strictly greater than the profit from setting $p_{1}=s_{2}$ and sharing demand with Firm 2. As above, we deal with this situation in a limiting sense and simply use $s_{2}$ in place of $s_{2}-\epsilon$, 
where it is understood that Firm 1 serves the entire market. Hence,

$$
p_{1}^{\star}=\min \left(s_{2}, p_{M}^{\star}\right),
$$

and

$$
p^{\star}=\left(p_{1}^{\star}, s_{2}, s_{3}, \ldots, s_{N}\right) .
$$

Expression (60) is the analog of the Type $\langle\mathrm{III}\rangle$ equilibrium from the differentiated Bertrand game. Firm 2 can be on the boundary, with Firm 1 pricing below his monopoly price because they must respect the presence of Firm 2. However, if the cost of Firm 2 is high enough, then Firm 1 can completely ignore the higher cost firms and we are in the setting analogous to a Type $\langle$ II $\rangle$ equilibrium.

The only subtlety that arises in this case is what occurs when firms have equal cost. This is only relevant if the firms that are tied also have the lowest costs in the market. Suppose for some $k>1$ we have $s_{1}=s_{2}=\cdots=s_{k}$ and $s_{k}<s_{k+1} \leq s_{k+2} \leq \cdots \leq s_{N}$. Due to the potential for undercutting, the only equilibrium is for all firms to set price equal to marginal cost. This can be seen by comparing the profit at any other equilibrium price and that obtained by a single firm deviating slightly and capturing the entire market. Hence,

$$
p^{\star}=\left(s_{1}, \ldots, s_{1}, s_{k+1}, \ldots, s_{N}\right),
$$

where it is understood that Firms 1 through $k$ split demand equally and the remaining firms get zero demand and make zero profit.

\section{References}

[1] R. Amir and J. Y. Jin. Cournot and Bertrand equilibria compared: substitutability, complementarity and concavity. Int. J. Ind. Organ., 19(3-4):303 - 317, 2001.

[2] R. Amir and V. Lambson. Entry, exit, and imperfect competition in the long run. J. Econ. Theory, 110(1):191 - 203, 2003.

[3] J. Bertrand. Théorie mathématique de la richesse sociale. Journal des Savants, 67:499-508, 1883.

[4] R. Caldentey and M. Haugh. A Cournot-Stackelberg Model of Supply Contracts with Financial Hedging. Working paper, NYU Stern School of Business, 2010.

[5] R. Cellini, L. Lambertini, and G. Ottaviano. Welfare in a differentiated oligopoly with free entry: a cautionary note. Research in Economics, 58:125-133, 2004.

[6] A. Cournot. Recherches sur les Principes Mathématique de la Théorie des Richesses. Hachette, Paris, 1838. English translation by N.T. Bacon, published in Economic Classics, Macmillan, 1897, and reprinted in 1960 by Augustus M. Kelley.

[7] K. G. Dastidar. On the existence of pure strategy Bertrand equilibrium. Econ. Theory, 5:19-32, 1995.

[8] F. Edgeworth. Me teoria pura del monopolio. Giornale degli Economisti, 40:13-31, 1897. English translation: The pure theory of monopoly. In Papers Relating to Political Economy, Vol. I, edited by F. Edgeworth. London: Macmillan, 1925.

[9] W. Elberfeld and E. Wolfstetter. A dynamic model of Bertrand competition with entry. Int. J. Ind. Organ., 17(4):513 - 525, 1999. 
[10] J. Friedman. Oligopoly Theory. Cambridge University Press, 1983.

[11] B. Golany and U. G. Rothblum. Optimal investment in development projects. Operations Research Letters, 36:657-661, 2008.

[12] O. Guéant, J.-M. Lasry, and P.-L. Lions. Mean field games and oil production. Technical report, College de France, Paris, 2010.

[13] C. Harris, S. Howison, and R. Sircar. Games with exhaustible resources. SIAM J. Applied Mathematics, $70: 2556-2581,2010$.

[14] S. H. Hoernig. Bertrand games and sharing rules. Econ. Theory, 31:573-585, 2007.

[15] H. Hotelling. Stability in competition. Econ. J., 39(153):41-57, 1929.

[16] H. Hotelling. The economics of exhaustible resources. Journal of Political Economy, 39 (2):137-175, 1931.

[17] J. Jurgan. Cost variations in a differentiated good oligopoly. Working Papers 069, Bavarian Graduate Program in Economics (BGPE), February 2009.

[18] D. M. Kreps and J. A. Scheinkman. Quantity precommitment and Bertrand competition yield Cournot outcomes. Bell J. Econ., 14(2):326-337, 1983.

[19] A. Ledvina and R. Sircar. Dynamic Bertrand oligopoly. Applied Mathematics and Optimization, 63: $11-44,2011$.

[20] M. Ludkovski and R. Sircar. Exploration and exhaustibility in dynamic Cournot games. European J. Applied Mathematics, 2011. In press.

[21] A. Mas-Colell, M. D. Whinston, and J. R. Green. Microeconomic Theory. Oxford University Press, New York, New York, 1995.

[22] A. Mukherjee. Price and quantity competition under free entry. Research in Economics, 59:335-344, 2005.

[23] N. Singh and X. Vives. Price and quantity competition in a differentiated duopoly. RAND J. Econ., 15(4):546-554, 1984.

[24] X. Vives. Oligopoly pricing: old ideas and new tools. MIT Press, Cambridge, MA, 1999.

[25] P. Zanchettin. Differentiated duopoly with asymmetric costs. J. Econ. Manage. Strategy, 15:999-1015, 2006. 